\title{
Systematic study of the effects of lowering low-density lipoprotein-cholesterol on regression of coronary atherosclerotic plaques using intravascular ultrasound
}

Wen-Qian Gao ${ }^{1,2+}$, Quan-Zhou Feng ${ }^{1 *+}$, Yu-Feng Li ${ }^{1 \dagger}$, Yuan-Xin Li ${ }^{3}$, Ya Huang ${ }^{1}$, Yan-Ming Chen ${ }^{1}$, Bo Yang ${ }^{1}$ and Cai-Yi Lu' ${ }^{1 *}$

\begin{abstract}
Background: Conflicting results currently exist on the effects of LDL-C levels and statins therapy on coronary atherosclerotic plaque, and the target level of LDL-C resulting in the regression of the coronary atherosclerotic plaques has not been settled.

Methods: PubMed, EMBASE, and Cochrane databases were searched from Jan. 2000 to Jan. 2014 for randomized controlled or blinded end-points trials assessing the effects of LDL-C lowering therapy on regression of coronary atherosclerotic plaque (CAP) in patients with coronary heart disease by intravascular ultrasound. Data concerning the study design, patient characteristics, and outcomes were extracted. The significance of plaques regression was assessed by computing standardized mean difference (SMD) of the volume of CAP between the baseline and follow-up. SMD were calculated using fixed or random effects models.

Results: Twenty trials including 5910 patients with coronary heart disease were identified. Mean lowering LDL-C by $45.4 \%$ and to level $66.8 \mathrm{mg} / \mathrm{dL}$ in the group of patients with baseline mean LDL-C $123.7 \mathrm{mg} / \mathrm{dL}$, mean lowering LDL-C by $48.8 \%$ and to level $60.6 \mathrm{mg} / \mathrm{dL}$ in the group of patients with baseline mean LDL-C $120 \mathrm{mg} / \mathrm{dL}$, and mean lowering LDL-C by $40.4 \%$ and to level $77.8 \mathrm{mg} / \mathrm{dL}$ in the group of patients with baseline mean LDL-C $132.4 \mathrm{mg} / \mathrm{dL}$ could significantly reduce the volume of CAP at follow up (SMD $-0.108 \mathrm{~mm}^{3}, 95 \% \mathrm{Cl}-0.176 \sim-0.040, p=0.002$; $\mathrm{SMD}-0.156 \mathrm{~mm}^{3}, 95 \% \mathrm{Cl}-0.235 \sim-0.078, p=0.000 ; \mathrm{SMD}-0.123 \mathrm{~mm}^{3}, 95 \% \mathrm{Cl}-0.199 \sim-0.048, p=0.001$; respectively). LDL-C lowering by rosuvastatin (mean $33 \mathrm{mg}$ daily) and atorvastatin (mean $60 \mathrm{mg}$ daily) could significantly decrease the volumes of CAP at follow up (SMD $-0.162 \mathrm{~mm}^{3}, 95 \% \mathrm{Cl}:-0.234 \sim-0.081, p=0.000$; SMD -0.101 , 95\% Cl: $-0.184 \sim-0.019, p=0.016$; respectively). The mean duration of follow up was from $17 \sim 21$ months.

Conclusions: Intensive lowering LDL-C (rosuvastatin mean $33 \mathrm{mg}$ daily and atorvastatin mean $60 \mathrm{mg}$ daily) with $>17$ months of duration could lead to the regression of CAP, LDL-C level should be reduced by $>40 \%$ or to a target level $<78 \mathrm{mg} / \mathrm{dL}$ for regressing CAP.
\end{abstract}

Keywords: Low-density lipoprotein-cholesterol, Coronary atherosclerotic plaque, Intravascular ultrasound, Coronary artery disease

\footnotetext{
*Correspondence: fqz301@yahoo.com; cylu2000@126.com

${ }^{\dagger}$ Equal contributors

'The Department of Cardiology, Chinese PLA General Hospital, Beijing 100853, China

Full list of author information is available at the end of the article
} 


\section{Background}

It is universally accepted that high serum concentrations of low-density lipoprotein cholesterol (LDL-C) can lead to atherosclerosis and accelerate the progression of atherosclerosis which is main causes of coronary artery disease [1]. Disruption of coronary atherosclerotic plaque (CAP) with subsequent thrombus formation may lead to sudden cardiac death, acute myocardial infarction, or unstable angina [2]. The evidence showed that reducing LDL-C can prevent coronary heart disease (CHD) and improve survival of $\mathrm{CHD}$ based on results from multiple randomized controlled trials (RCTs) [3,4].

For many years coronary angiography (CAG) has been the gold standard method for the investigation of the anatomy of coronary arteries and measure the efficacy of anti-atherosclerotic drug therapies $[5,6]$. But changes in CAG are measured only in the vascular lumen and not in the vessel wall [7], where the atherosclerotic process is located. Intravascular ultrasound (IVUS) is superior to angiography in the detection of early plaque formation and changes in plaque volume [8-10]. Through IVUS, Takagi et al. found that pravastatin lowered serum cholesterol levels and reduced the progression of CAP in patients with elevated serum cholesterol levels in 1997 [11]. Since then, multiple RCTs and no RCT about the effect of lowering LDL-C therapy on the regression of coronary atherosclerosis have been performed [12-16]. But the results varied with the RCTs: intensive LDL-C lowering therapy could reduce the progression of the plaques [12]; the mild LDL-C lowering did not [14-16]. The meta-analysis by Bedi et al. [17] evaluated the effects of LDL-C lowering on CAP by comparing statins with control therapy, and demonstrated that treatment with statins could slow atherosclerotic plaque progression and lead to plaque regression. The meta-analysis by Tian et al. [18] showed that CAP could be regressed in group of patients with $<100 \mathrm{mg}$ of LDL-C level at follow up. But so far, there are no systematic reviews of the effects of LDL-C levels on CAP, and the targets of LDL-C level that could result in the regression of the plaques have not been settled.

In this study, we conducted meta-analyses to summarize findings from the current trials on LDL-C lowering therapy retarding the progression of the CAP and to identify the targets of LDL-C resulting in the regression of the CAP for guiding the LDL-C lowering therapy. Effect of different statins on the progression of the CAP was also investigated.

\section{Methods}

\section{Search strategy and selection criteria}

An electronic literature search was performed to identify all relevant studies published in PubMed, EMBASE, and Cochrane databases in the English language from Jan. 1,
2000 to Jan. 1, 2014, using the terms "atherosclerosis" and "cholesterol blood level". The references of the studies were also searched for relevant studies. Studies were included using the following criteria: 1) randomized controlled or prospective, blinded end-points trials in which patients with CHD were assigned to LDL-C lowering therapy or placebo, and its primary end point was CAP change detected by IVUS; 2) report of LDL-C levels at baseline and follow-up (in each arm) or the level of LDL-C which can be calculated from the data in the paper (as in the trial by Yokoyama M [15], in which the LDL-C concentrations in control arm were directly extracted from the figure); 3) data on the volume of CAP, detected in IVUS at baseline and follow-up (in each arm), and volume of CAP was calculated as vessel volume minus lumen volume; Exclusion criteria were: 1) only CAP area or volume index or percent atheroma volume were detected by IVUS; 2) the levels of LDL-C at baseline or follow-up were not provided; and 3) target plaques were unstable.

\section{Data extraction and quality assessment}

Two investigators independently reviewed all potentially eligible studies and collected data on patient and study characteristics (author, year, design, sample size, the measures of LDL-C lowering, LDL-C levels, follow-up duration, and plaque volume), and any disagreement was resolved by consensus. The primary end point of this study was progression or regression of CAP detected by IVUS. Quality assessments were evaluated with Jadad quality scale [19].

\section{Data synthesis and analysis}

Continuous variables (change of CAP volume from baseline to follow-up) were analyzed using standardized mean differences (SMD).

The trials may have control arm and multiple active treatment arms, changes of plaque volume in every arms were used for pooled analysis. According to the levels and the reducing percentage of LDL-C at follow-up, the arms were grouped to following groups: $\leq 70,>70 \leq$ $100 \mathrm{HP}$ ( $>70 \leq 100 \mathrm{mg}$ and reducing percentage $\geq 30 \%$ ), $>70 \leq$ 100MP $(>70 \leq 100 \mathrm{mg}$ and reducing percentage $\geq 0<$ $30 \%),>70 \leq 100 \mathrm{LP}(>70 \leq 100 \mathrm{mg}$ and reducing percentage $<0 \%$ ), $>100 \mathrm{mg} / \mathrm{dL}$; and $<0, \geq 0<30, \geq 30<40, \geq 40<$ $50, \geq 50 \%$ respectively, to investigate the effect of different levels of LDL-C at follow up on CAPs. According to different statins, the arms were grouped to following groups: rosuvastatin, atorvastatin, pitavastatin, simvastatin, fluvastatin and pravastatin group, to investigate the effect of different statins on CAPs. The volume of CAP at follow up was compared with that at baseline to evaluate effect of LDL-C levels on regression of CAP. 
Heterogeneity across trials (arms) was assessed via a standard $\chi^{2}$ test with significance being set at $p<0.10$ and also assessed by means of $I^{2}$ statistic with significance being set at $I^{2}>50 \%$. Pooled analyses were calculated using fixed-effect models, whereas random-effect models were applied in case of significant heterogeneity across studies (arms). Sensitivity analyses (exclusion of one study at one time) were performed to determine the stability of the overall effects of LDL-C levels. Additionally, publication bias was assessed using the Egger regression asymmetry test. Mean LDL-C level and follow up duration of groups were calculated by descriptive statistics. A two-sided $p$ values $<0.05$ was considered statistically significant. Statistical analyses were performed using STATA software 12.0 (StataCorp, College Station, Texas) and Review Manager V5.2 (Copenhagen: The Nordic Cochrane Centre, The Cochrane Collaboration, 2012).

\section{Results}

\section{Eligible studies}

The flow of selecting studies for the meta-analysis is shown in Figure 1. Briefly, of the initial 647 articles, one hundred and twenty of abstracts were reviewed, resulting in exclusion of 100 articles, and 20 articles were reviewed in full text, resulting in exclusion of 10 trials and inclusion of 18 additional trials. Twenty two RCTs [12-16,20-31], [32-36] and six blinded end-points trial [37-42] were carefully evaluated. Five trials were excluded because of specific the index of plaque (volume index in TRUTH [24], trial by Kovarnik $\mathrm{T}$ [31], by Hattori K [42], and by Petronio AS [32]; area in LACMART [38]); GAIN [20] excluded because of no data of plaque volume at follow up; trial by Zhang X [25] excluded because of no data of LDL-C; trial by Hong YJ [30] excluded because of wrong data at follow up. Sixteen RCT (ESTABLISH [14], REVERSAL [13], A-PLUS [21], ACTIVATE

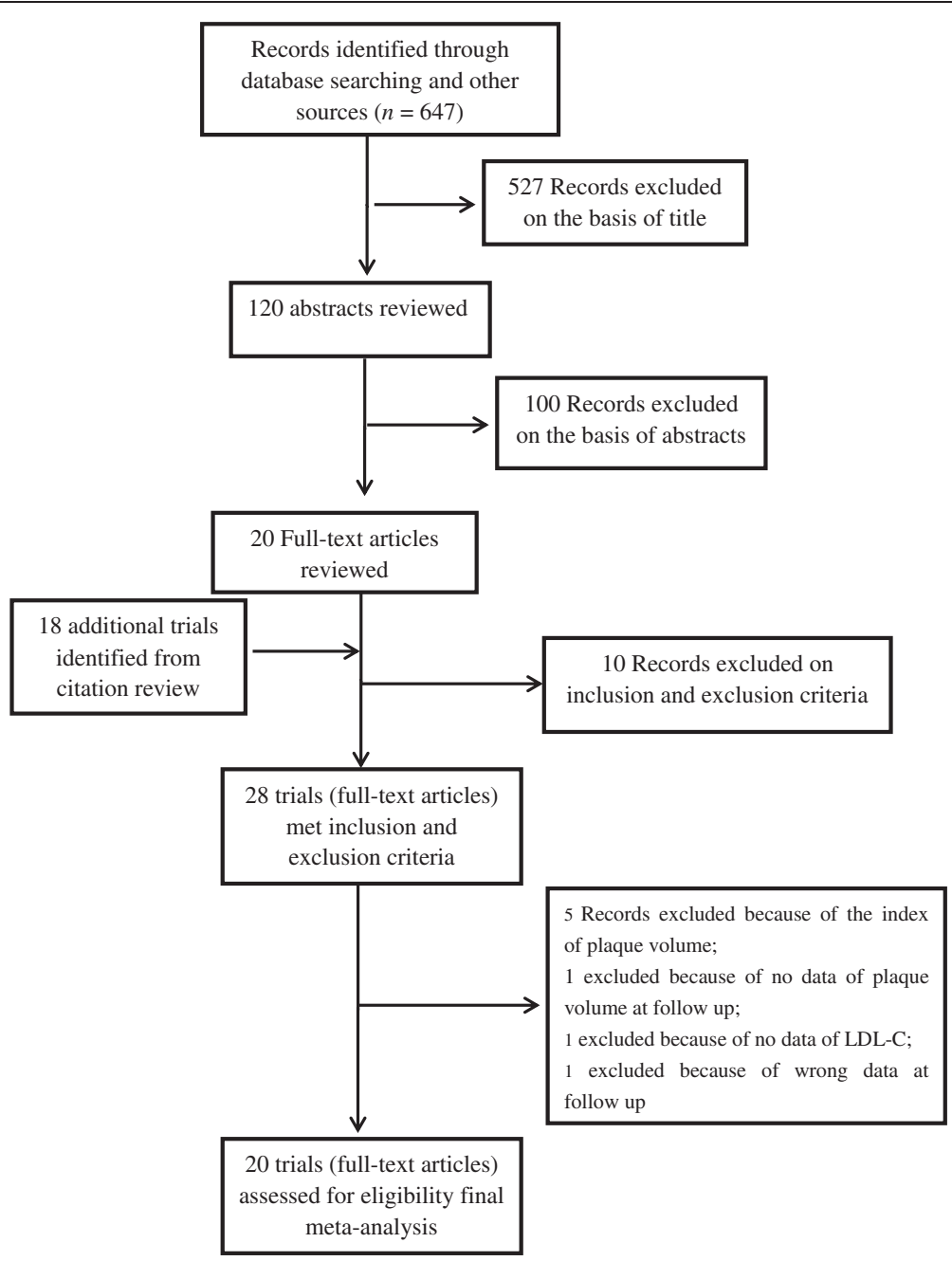

Figure 1 Flow diagram of study-screening process. 
[22], ILLUSTRATE [23], JAPAN-ACS [12], REACH [26], SATURN [28], ARTMAP [29], ERASE [34], STRADIVARIUS [35], PERISCOPE [36], and trials by Yokoyama M [15], by Kawasaki M [16], by Hong MK [27], and Tani S [33]) and four blinded end-points trial (ASTEROID [37], COSMOS [40], trial by Jensen LO [39] and trial by Nasu $\mathrm{K}$ [41]) were finally analyzed.

The characteristics of the included trials were shown in Table 1. Among the 20 trials, there were 15 trials assessing statins (statin vs. usual care in 6 trials [14-16,26,33,41]; intensive statin vs. moderate statin treatment in 5 trials [12,13,27-29]; follow up vs baseline in 3 trial $[37,39,40]$, before acute coronary syndrome (ACS) vs after ACS in one trial [34]), 2 trials assessing enzyme acyl-coenzyme A: cholesterol acyltransferase (ACAT) inhibition (vs. placebo, both on the basis of mean LDL-C $<102$ after background lipid-lowering therapy with statins in $62-79 \%$ of patients) [21,22], one trial assessing cholesteryl ester transfer protein (CETP) inhibitor torcetrapib (vs. statins on the basis of LDL-C $\leq$ 100 by statins) [23], one trial assessing a decreasing obesity drug: rimonabant (vs. placebo, on the basis of statins therapy) [35], and one trial assessing glucoselowering agents (pioglitazone vs glimepiride on the basis of statins therapy) [36]. In three trials [12,14,34] with acute coronary syndrome, all target plaques were selected in non-culprit vessels. Overall, 5910 patients with CHD underwent serial IVUS examination for evaluating regression of CAP. Follow-up periods ranged from 2 to 24 months. The levels of LDL-C of each arm at baseline and follow-up were shown in Table 2.

Risk of bias of included studies, evaluated through Cochrane's methods, showed an overall acceptable quality of selected trials (Figures 2 and 3 ).

\section{The effect of the levels of LDL-C at follow-up on regression of coronary atherosclerotic plaque}

LDL-C lowering in group $\leq 70$ and $>70 \leq 100 \mathrm{HP} \mathrm{mg} / \mathrm{dL}$ could lead to regression of CAP, but LDL-C lowering in group $>70 \leq 100 \mathrm{MP},>70 \leq 100 \mathrm{LP}$ and $>100 \mathrm{mg} / \mathrm{dL}$ could not (Figure 4, Table 3).

In group $\leq 70 \mathrm{mg} / \mathrm{dL}$ (including seven arms) with mean 18.6 months of follow up and group $>70 \leq 100 \mathrm{HP}$ $\mathrm{mg} / \mathrm{dL}$ (including eleven arms) with mean 17.4 months of follow up, the volumes of CAP $\left(125.9,123.8 \mathrm{~mm}^{3}\right.$ respectively) at follow up were significantly decreased, compared with the volumes $\left(177.1,129.7 \mathrm{~mm}^{3}\right.$ respectively) at baseline [SMD $-0.156 \mathrm{~mm}^{3}, 95 \%$ CI (confidence interval) $-0.235 \sim-0.078, p=0.000$; SMD $-0.123 \mathrm{~mm}^{3}$, $95 \%$ CI $-0.199 \sim-0.048, p=0.001$; respectively]. There was no significant heterogeneity among arms ( $\chi^{2}$ for heterogeneity $=0.57, p=0.997, I^{2}=0 \%$ for group $\leq 70 \mathrm{mg} / \mathrm{dL} ; X^{2}$ for heterogeneity $=6.83, p=0.741, I^{2}=0 \%$ for group $>70 \leq$ $100 \mathrm{HP} \mathrm{mg} / \mathrm{dL})$.
Sensitivity analyses suggested that LDL-C lowering in group $\leq 70$ and $>70 \leq 100 \mathrm{HP} \mathrm{mg} / \mathrm{dL}$ could lead to regression of CAP with reduction of the CAP volume ranged from $-0.146 \mathrm{~mm}^{3}$ (SMD, 95\% CI: $-0.238 \sim-0.054$ ) when the arm of 2006 ASTEROID Ros was omitted to $-0.167 \mathrm{~mm}^{3}$ (SMD, 95\% CI: $-0.270 \sim-0.064$ ) when the arm of 2011 SATURN Ros was omitted; and from $-0.103 \mathrm{~mm}^{3}$ (SMD, 95\% CI: -0.182 -0.024) when the arm of 2009 JAPAN-ACS Ato was omitted to $-0.151 \mathrm{~mm}^{3}$ (SMD, 95\% CI: $-0.235 \sim-0.067$ ) when the arm of 2004 REVERSAL Ato was omitted. No publication bias was found, the values of $p$ by Egger's test for group $\leq 70$ and $>70 \leq 100 \mathrm{HP} \mathrm{mg} / \mathrm{dL}$ were $0.835,0.501$ respectively.

In group $>100 \mathrm{mg} / \mathrm{dL}$ (including eleven arms) with mean 14.6 months of follow up, the volume of CAP at follow up was not significantly increased, compared with the volumes at baseline (SMD $0.013 \mathrm{~mm}^{3}, 95 \%$ CI -0.092 $0.118, p=0.809$ ). There was no significant heterogeneity among arms ( $\chi^{2}$ for heterogeneity $=2.49, p=0.991, I^{2}=0 \%$ ).

Sensitivity analyses suggested that LDL-C lowering to $>100 \mathrm{mg} / \mathrm{dL}$ at follow-up could still not lead to regression of CAP with reduction of the plaque volume ranged from $-0.005 \mathrm{~mm}^{3}$ (95\% CI $-0.136 \sim 0.126$ ) when the arm of 2004 REVERSAL Pro was omitted to $0.034 \mathrm{~mm}^{3}$ (SMD, 95\% CI -0.075 0.143) when 2005 Tani S Pra was omitted. No publication bias was observed from the values of $p$ (0.566) by Egger's test.

Mean levels of LDL-C at baseline and follow up and mean reducing percentage of LDL-C in group $\leq 70,>70 \leq$ $100 \mathrm{HP},>70 \leq 100 \mathrm{MP},>70 \leq 100 \mathrm{LP}$ and $>100 \mathrm{mg} / \mathrm{dL}$ were showed in Table 4.

\section{The effect of the LDL-C reducing percentage at follow-up on regression of CAP}

LDL-C lowering in group $\geq 30<40, \geq 40<50, \geq 50 \%$ could lead to regression of CAP, but LDL-C lowering in group $<0$ and $\geq 0<30 \%$ could not (Figure 5 , Table 3 ).

In group $\geq 30<40 \%$ (including ten arms) with mean 10.3 months of follow up, and group $\geq 40<50 \%$ (including eight arms) with mean 19.4 months of follow up, the volumes of CAP $\left(94.3,150.7 \mathrm{~mm}^{3}\right.$ respectively) at follow up were significantly decreased, compared with the volumes $\left(102.9,157.8 \mathrm{~mm}^{3}\right.$ respectively) at baseline (SMD $-0.199 \mathrm{~mm}^{3}, 95 \% \mathrm{CI}-0.314 \sim-0.085, p=0.001$; SMD $-0.108 \mathrm{~mm}^{3}$, 95\% CI $-0.176 \sim-0.040, p=0.002$; respectively). There was no significant heterogeneity among arms $\left(\chi^{2}\right.$ for heterogeneity $=3.10, P=0.960, I^{2}=$ $0 \%$; $\chi^{2}$ for heterogeneity $=2.50, p=0.927, I^{2}=0 \%$; for group $\geq 30<40$, and group $\geq 40<50$ respectively).

Sensitivity analyses showed that LDL-C lowering in group $\geq 30<40 \%$ and group $\geq 40<50$ could still lead to regression of CAP with reduction of the plaque volume ranged from $-0.166 \mathrm{~mm}^{3}$ (95\% CI $\left.-0.295 \sim-0.038\right)$ when the arm of 2009 JAPAN-ACS Ato was omitted 
Table 1 Features of participating trials

\begin{tabular}{|c|c|c|c|c|c|c|c|c|c|c|}
\hline $\begin{array}{l}\text { Authors and } \\
\text { trial name }\end{array}$ & Trial type and location & Objective & Year & $\mathrm{N} \mathrm{T/C}$ & $\begin{array}{l}\text { Study } \\
\text { population }\end{array}$ & $\begin{array}{l}\text { LDL-C at } \\
\text { follow up }\end{array}$ & $\begin{array}{l}\mathrm{LDL}-\mathrm{C} \\
\text { reducing } \\
\text { percentage }\end{array}$ & Treatments & Follow up & Main Results or Conclusion \\
\hline $\begin{array}{l}\text { Okazaki S [14]; } \\
\text { ESTABLISH }\end{array}$ & $\begin{array}{l}\text { RCT: prospective, open-label, } \\
\text { randomized, single center } \\
\text { study. Japan }\end{array}$ & $\begin{array}{l}\text { Effects of statins on } \\
\text { changes in plaque } \\
\text { by IVUS }\end{array}$ & 2004 & $24 / 24$ & ACS & $70 / 119$ & $-44 /-0.004$ & Ato 20 vs Diet & 6 & $\begin{array}{l}\text { Plaque volume was sigificantly } \\
\text { reduced in the Ato group } \\
\text { compared with the control } \\
\text { group. }\end{array}$ \\
\hline $\begin{array}{l}\text { Nissen SE [13]; } \\
\text { REVERSAL }\end{array}$ & $\begin{array}{l}\text { RCT: Double-blind, } \\
\text { randomized active } \\
\text { control multicenter } \\
\text { trial; USA }\end{array}$ & $\begin{array}{l}\text { Effects of statins } \\
\text { (intensive or } \\
\text { moderate) on } \\
\text { changes in plaque } \\
\text { by IVUS }\end{array}$ & 2004 & $253 / 249$ & CAD & $79 / 110$ & $-46 /-25$ & Ato 80 vs Pra40 & 18 & $\begin{array}{l}\text { Ato reduced progression of } \\
\text { coronary plaque compared } \\
\text { with Pra. Compared with } \\
\text { baseline values, Ato had no } \\
\text { change in atheroma burden, } \\
\text { whereas patients treated } \\
\text { with Pra showed progression } \\
\text { of coronary plaque. }\end{array}$ \\
\hline $\begin{array}{l}\text { Tardif JC [21]; } \\
\text { A-PLUS }\end{array}$ & $\begin{array}{l}\text { RCT: international, } \\
\text { multicenter, double-blind, } \\
\text { placebo-controlled, } \\
\text { randomized trial. Canada, } \\
\text { USA }\end{array}$ & $\begin{array}{l}\text { Effects of different } \\
\text { dosage of avasimibe } \\
\text { on changes in plaque } \\
\text { by IVUS }\end{array}$ & 2004 & $\begin{array}{l}108 / 98 / \\
117 / 109\end{array}$ & CAD & $\begin{array}{l}100 / 102 / \\
101 / 91\end{array}$ & $\begin{array}{l}7.8 / 9.1 / \\
10.9 / 1.7\end{array}$ & $\begin{array}{l}\text { Ava50, } 250 \text {, and } \\
750 \text { vs Placebo } \\
\text { on the basis of } \\
\text { LDL-C }<125\end{array}$ & 18 & $\begin{array}{l}\text { Avasimibe did not favorably } \\
\text { alter coronary atherosclerosis } \\
\text { as assessed by IVUS. }\end{array}$ \\
\hline Jensen LO [39] & $\begin{array}{l}\text { Open non placebo } \\
\text { controlled serial investigation; } \\
\text { blinded end-points. Denmark }\end{array}$ & $\begin{array}{l}\text { To investigate the } \\
\text { effect of lipid lowering } \\
\text { by simvastatin on } \\
\text { coronary atherosclerotic } \\
\text { plaque volumes and } \\
\text { lumen. }\end{array}$ & 2004 & 40 & CAD & 85 & -46.3 & $\operatorname{Sim} 40$ & 15 & $\begin{array}{l}\text { Lipid-lowering therapy with } \\
\text { Sim is associated with a } \\
\text { significant plaque regression } \\
\text { in coronary arteries. }\end{array}$ \\
\hline Yokoyama M [15] & $\begin{array}{l}\text { RCT: randomized, single } \\
\text { center. Japan }\end{array}$ & $\begin{array}{l}\text { Effects of statins on } \\
\text { changes in plaque } \\
\text { by IVUS }\end{array}$ & 2005 & $29 / 30$ & $\begin{array}{l}\text { Stable } \\
\text { angina }\end{array}$ & $87 / 124$ & $-35 /-0.075$ & Ato 10 vs Diet & 6 & $\begin{array}{l}\text { Treatment with Ato may } \\
\text { reduce volumes of coronary } \\
\text { plaques. }\end{array}$ \\
\hline Kawasaki M [16] & $\begin{array}{l}\text { RCT: randomization, } \\
\text { open-label, single-center } \\
\text { study. Japan }\end{array}$ & $\begin{array}{l}\text { Effects of statins on } \\
\text { changes in plaque } \\
\text { by IVUS }\end{array}$ & 2005 & $17 / 18 / 17$ & $\begin{array}{l}\text { Stable } \\
\text { angina }\end{array}$ & $95 / 102 / 149$ & $-39 /-32 /-0.02$ & $\begin{array}{l}\text { Ato } 20 \text {, Pra } \\
20 \text { vs Diet }\end{array}$ & 6 & $\begin{array}{l}\text { Treatment with Ato and Pra } \\
\text { may not significantly reduce } \\
\text { volumes of coronary plaques. }\end{array}$ \\
\hline Tani S [33] & $\begin{array}{l}\text { RCT: a prospective, } \\
\text { single-center, randomized, } \\
\text { open trial. Japan }\end{array}$ & $\begin{array}{l}\text { Investigated the effects } \\
\text { of pravastatin on the } \\
\text { serum levels of } \\
\text { MDA-LDL and coronary } \\
\text { atherosclerosis. }\end{array}$ & 2005 & $52 / 23$ & $\begin{array}{l}\text { Stable } \\
\text { angina }\end{array}$ & $104 / 120$ & $-20 /-2.4$ & Pra $10-20$ vs con & 6 & $\begin{array}{l}\text { Plaque volume was sigificantly } \\
\text { reduced in the Pra group } \\
\text { compared with the control } \\
\text { group. }\end{array}$ \\
\hline $\begin{array}{l}\text { Nissen SE [22]; } \\
\text { ACTIVATE }\end{array}$ & $\begin{array}{l}\text { RCT: randomized, } \\
\text { multicenter. USA }\end{array}$ & $\begin{array}{l}\text { Effects of pactimibe } \\
\text { on changes in plaque } \\
\text { by IVUS }\end{array}$ & 2006 & $206 / 202$ & CAD & $91 / 86$ & $-9.6 /-14.9$ & Pac100 vs Placebo & 18 & $\begin{array}{l}\text { Pac is not an effective } \\
\text { strategy for limiting } \\
\text { atherosclerosis and may } \\
\text { promote atherogenesis. }\end{array}$ \\
\hline
\end{tabular}


Table 1 Features of participating trials (Continued)

\begin{tabular}{|c|c|c|c|c|c|c|c|c|c|c|}
\hline $\begin{array}{l}\text { Nissen SE [37]; } \\
\text { ASTEROID }\end{array}$ & $\begin{array}{l}\text { Prospective, open-label } \\
\text { blinded end-points. USA, } \\
\text { Germany, France, Canada }\end{array}$ & $\begin{array}{l}\text { Effects of Statins with } \\
\text { different levels of } \\
\text { LDL-C on changes } \\
\text { in plaque by IVUS }\end{array}$ & 2006 & 349 & CAD & 61 & -53.2 & Ros 40 & 24 & $\begin{array}{l}\text { Therapy using Ros can } \\
\text { result in significant } \\
\text { regression of atherosclerosis. }\end{array}$ \\
\hline $\begin{array}{l}\text { Yamada T [26]; } \\
\text { REACH }\end{array}$ & $\begin{array}{l}\text { RCT: open-labeled, } \\
\text { randomized, multicenter } \\
\text { study. Japan }\end{array}$ & $\begin{array}{l}\text { Evaluate the effect } \\
\text { of marked reduction } \\
\text { of } L D L-C \text { in patients } \\
\text { with } C H D \text { on } \\
\text { progression of } \\
\text { atherosclerosis. }\end{array}$ & 2007 & $26 / 32$ & $\begin{array}{l}\text { Stable } \\
\text { angina }\end{array}$ & $83 / 115$ & $-43 / 0$ & Ato 5 vs Con & 12 & $\begin{array}{l}\text { Ato treatment prevented } \\
\text { the further progression of } \\
\text { atherosclerosis by } \\
\text { maintaining LDL-C below } \\
100 \mathrm{mg} / \mathrm{dl} \text { in patients with } \\
\mathrm{CHD} \text {. }\end{array}$ \\
\hline $\begin{array}{l}\text { Nissen SE [23]; } \\
\text { ILLUSTRATE }\end{array}$ & $\begin{array}{l}\text { RCT: prospective, randomized, } \\
\text { multicenter, double-blind } \\
\text { clinical trial. North America } \\
\text { or Europe }\end{array}$ & $\begin{array}{l}\text { Effects of CETP } \\
\text { inhibitor on changes } \\
\text { in plaque by IVUS }\end{array}$ & 2007 & $446 / 464$ & CAD & $87 / 70$ & 6.6/-13.3 & $\begin{array}{l}\text { Ato } 10-80 \text { vs Ato+Tor } \\
60 \text { on the basis of } \\
\text { LDL-C } \leq 100 \text { by Ato }\end{array}$ & 24 & $\begin{array}{l}\text { The Tor was associated } \\
\text { with a substantial increase } \\
\text { in } \mathrm{HDL}-\mathrm{C} \text { and decrease in } \\
\mathrm{LDL}-\mathrm{C} \text {, and there was no } \\
\text { significant decrease in the } \\
\text { progression of coronary } \\
\text { atherosclerosis. }\end{array}$ \\
\hline $\begin{array}{l}\text { Nissen SE [36]; } \\
\text { PERISCOPE }\end{array}$ & $\begin{array}{l}\text { RCT: prospective, randomized, } \\
\text { multicenter, double-blind } \\
\text { clinical trial. USA }\end{array}$ & $\begin{array}{l}\text { To compare the } \\
\text { effects of pioglitazone, } \\
\text { and glimepiride on } \\
\text { the progression of } \\
\text { coronary atherosclerosis } \\
\text { in patients with type } \\
2 \text { diabete and CAD }\end{array}$ & 2008 & $181 / 179$ & $C A D, D M$ & $96.1 / 95.6$ & $1.8 / 2.2$ & $\begin{array}{l}\text { Gli1-4 mg vs Pio } \\
15-45 \mathrm{mg} \text { on } \\
\text { bases of statins } \\
\text { therapy }\end{array}$ & 18 & $\begin{array}{l}\text { In patients with type } 2 \\
\text { diabetes and CAD, } \\
\text { treatment with Pio resulted } \\
\text { in a significantly lower rate } \\
\text { of progression of coronary } \\
\text { atherosclerosis compared } \\
\text { with Gli. }\end{array}$ \\
\hline $\begin{array}{l}\text { Nissen SE [35]; } \\
\text { STRADIVARIUS }\end{array}$ & $\begin{array}{l}\text { RCT: Randomized, } \\
\text { double-blinded, } \\
\text { placebo-controlled, 2-group, } \\
\text { parallel-group trial. North } \\
\text { America, Europe, and } \\
\text { Australia }\end{array}$ & $\begin{array}{l}\text { The effect of } \\
\text { rimonabant on } \\
\text { regression of coronary } \\
\text { disease in patients } \\
\text { with the metabolic } \\
\text { syndrome and CAD }\end{array}$ & 2008 & $335 / 341$ & $\begin{array}{l}\text { CAD, } \\
\text { Obesity }\end{array}$ & $87.6 / 86.3$ & $-4.7 /-3.6$ & $\begin{array}{l}\text { Rim } 20 \text { mg vs } \\
\text { Placebo on } \\
\text { bases of statins } \\
\text { therapy }\end{array}$ & 18 & $\begin{array}{l}\text { Rim can reduce progression } \\
\text { of coronary plaque, and } \\
\text { increase HDL-C levels, } \\
\text { decrease triglyceride levels. }\end{array}$ \\
\hline $\begin{array}{l}\text { Hiro T [12]; } \\
\text { JAPAN-ACS }\end{array}$ & $\begin{array}{l}\text { RCT: prospective, } \\
\text { randomized, open-label, } \\
\text { parallel group, multicenter. } \\
\text { Japan }\end{array}$ & $\begin{array}{l}\text { Effects of statins on } \\
\text { changes in plaque } \\
\text { by IVUS }\end{array}$ & 2009 & $127 / 125$ & ACS & $84 / 81$ & $-36 /-36$ & Ato 20 vs Pit 4 & 10 & $\begin{array}{l}\text { The administration of Pit ol } \\
\text { Ato in patients with ACS } \\
\text { equivalently resulted in } \\
\text { significant regression of } \\
\text { coronary plaque volume. }\end{array}$ \\
\hline $\begin{array}{l}\text { Takayama T; } \\
\text { COSMOS [40] }\end{array}$ & $\begin{array}{l}\text { Prospective, open-label } \\
\text { blinded end-points } \\
\text { multicenter trial. Japan }\end{array}$ & $\begin{array}{l}\text { Evaluate the effect } \\
\text { of rosuvastatin on } \\
\text { plaque volume in } \\
\text { patients with stable } \\
\text { CAD, including those }\end{array}$ & 2009 & 126 & $\begin{array}{l}\text { Stable } \\
\text { angina }\end{array}$ & 83 & -38.6 & $\operatorname{Ros}<20$ & 14 & $\begin{array}{l}\text { Ros exerted significant } \\
\text { regression of coronary } \\
\text { plaque volume in Japanese } \\
\text { patients with stable CAD. }\end{array}$ \\
\hline
\end{tabular}


Table 1 Features of participating trials (Continued)

\begin{tabular}{|c|c|c|c|c|c|c|c|c|c|c|}
\hline $\begin{array}{l}\text { Rodés-Cabau; } \\
\text { ERASE [34] }\end{array}$ & $\begin{array}{l}\text { RCT: multicenter randomized } \\
\text { placebo-controlled. Canada }\end{array}$ & $\begin{array}{l}\text { Evaluate the early } \\
\text { effects of newly } \\
\text { initiated statin therapy } \\
\text { on coronary } \\
\text { atherosclerosis as } \\
\text { evaluated by IVUS. }\end{array}$ & 2009 & $38 / 36$ & ACS & $77 / 63$ & $8.5 /-37$ & $\begin{array}{l}\text { Before ACS vs } \\
\text { After ACS }\end{array}$ & $<2$ & $\begin{array}{l}\text { Newly initiated statin } \\
\text { therapy is associated with } \\
\text { rapid regression of coronary } \\
\text { atherosclerosis. }\end{array}$ \\
\hline Nasu K [41] & $\begin{array}{l}\text { Prospective and multicenter } \\
\text { study with nonrandomized } \\
\text { and non-blinded design, } \\
\text { but blinded end. Japan }\end{array}$ & $\begin{array}{l}\text { Evaluate the effect } \\
\text { of treatment with } \\
\text { statins on the } \\
\text { progression of } \\
\text { coronary atherosclerotic } \\
\text { plaques of a nonculprit } \\
\text { vessel by serial IVUS. }\end{array}$ & 2009 & $40 / 39$ & $\begin{array}{l}\text { Stable } \\
\text { angina }\end{array}$ & $98.1 / 121$ & $-32.3 /-1.1$ & Flu 60 vs Con & 12 & $\begin{array}{l}\text { One-year lipid-lowering } \\
\text { therapy by Flu showed } \\
\text { significant regression of } \\
\text { plaque volume. }\end{array}$ \\
\hline Hong MK [27] & $\begin{array}{l}\text { RCT: randomized } \\
\text { control trial. Korea. }\end{array}$ & $\begin{array}{l}\text { Evaluated the effects } \\
\text { of statin treatments } \\
\text { for each component } \\
\text { of coronary plaques. }\end{array}$ & 2009 & $50 / 50$ & $\begin{array}{l}\text { Stable } \\
\text { angina }\end{array}$ & $78 / 64$ & $-34.5 /-44.8$ & Sim 20 vs Ros 10 & 12 & $\begin{array}{l}\text { Statin treatments } \\
\text { might be associated } \\
\text { with significant } \\
\text { changes in necrotic } \\
\text { core and fibrofatty } \\
\text { plaque volume. }\end{array}$ \\
\hline $\begin{array}{l}\text { Nicholls SJ; } \\
\text { SATURN [28] }\end{array}$ & $\begin{array}{l}\text { RCT: a prospective, } \\
\text { randomized, multicenter, } \\
\text { double-blind clinical trial. } \\
\text { USA }\end{array}$ & $\begin{array}{l}\text { Compare the effect } \\
\text { of these two intensive } \\
\text { statin regimens on } \\
\text { the progression of } \\
\text { coronary atherosclerosis. }\end{array}$ & 2011 & $519 / 520$ & CHD & $70.2 / 62.6$ & $-41.5 /-47.8$ & Ato 80 vs Ros 40 & 24 & $\begin{array}{l}\text { Maximal doses of Ros and } \\
\text { Ato resulted in significant } \\
\text { regression of coronary } \\
\text { atherosclerosis. }\end{array}$ \\
\hline $\begin{array}{l}\text { Lee CW [29]; } \\
\text { ARTMAP }\end{array}$ & $\begin{array}{l}\text { RCT: a prospective, } \\
\text { single-center, open-label, } \\
\text { randomized comparison } \\
\text { trial. Korea. }\end{array}$ & $\begin{array}{l}\text { Compared the effects } \\
\text { of atorvastatin } 20 \\
\text { mg/day versus } \\
\text { rosuvastatin } 10 \\
\text { mg/day on mild } \\
\text { coronary atherosclerotic } \\
\text { plaques. }\end{array}$ & 2012 & $143 / 128$ & $\begin{array}{l}\text { Stable } \\
\text { angina }\end{array}$ & $56 / 53$ & $-47 /-49$ & Ato 20 vs Ros 10 & 6 & $\begin{array}{l}\text { Usual doses of Ato and } \\
\text { Ros induced significant } \\
\text { regression of coronary } \\
\text { atherosclerosis in } \\
\text { statin-naive patients. }\end{array}$ \\
\hline
\end{tabular}

Abbreviations: RCT, randomized controlled trials; T, treatment group; C, control group IVUS, Intravascular ultrasound; CAD, Coronary artery disease; ACS, Acute coronary syndrome; CHD, Coronary heart disease; Ato, Atorvastatin; Ros, Rosuvastatin; Pra, Pravastatin; Pit, Pitavastatin; Sim, Simvastatin; Flu, Fluvastatin; Con, Control; Pac, Pactimibe; Tor, Torcetrapib, Ava 50, 250, 750, Avasimibe 50, 250, 750 mg; T/C, Treat/Control; Gli, Glimepiride; Pio, Pioglitazone; Rim, Rimonabant. 
Table 2 The levels of LDL-C at baseline and follow up in each arm of included trials

\begin{tabular}{|c|c|c|c|c|c|}
\hline \multirow{2}{*}{ Authors } & \multirow{2}{*}{ Trial name } & \multirow{2}{*}{$\begin{array}{l}\text { Management in } \\
\text { each arm }\end{array}$} & \multirow{2}{*}{$\mathbf{N}$} & \multicolumn{2}{|c|}{ LDL-C level } \\
\hline & & & & At Baseline & At Follow-up \\
\hline Tardif JC & A-PLUS & Avasimibe50 & 108 & $92.8 \pm 1.7$ & $100^{*}$ \\
\hline Tardif JC & A-PLUS & Avasimibe250 & 98 & $93.4 \pm 1.6$ & $101.9^{*}$ \\
\hline Tardif JC & A-PLUS & Avasimibe750 & 117 & $91.4 \pm 1.6$ & $101.4^{*}$ \\
\hline Tardif JC & A-PLUS & Placebo & 109 & $89.6 \pm 1.6$ & $91.1^{*}$ \\
\hline Okazaki S & ESTABLISH & Control & 24 & $123.9 \pm 35.3$ & $119.4 \pm 24.6$ \\
\hline Okazaki S & ESTABLISH & Atorvastatin & 24 & $124.6 \pm 34.5$ & $70.0 \pm 25.0$ \\
\hline Yokoyama M & & Control & 30 & $131.5 \pm 23 \#$ & $124.5 \pm 24.1 \#$ \\
\hline Yokoyama M & & Atorvastatin & 29 & $133 \pm 13$ & $87 \pm 29$ \\
\hline Nissen SE & REVERSAL & Atorvastatin & 253 & $150.2 \pm 27.9$ & $78.9 \pm 30.2$ \\
\hline Nissen SE & REVERSAL & Pravastatin & 249 & $150.2 \pm 25.9$ & $110.4 \pm 25.8$ \\
\hline Nissen SE & ACTIVATE & Pactimibe & 206 & $101.4 \pm 27.7$ & 91.3 \\
\hline Nissen SE & ACTIVATE & Placebo & 202 & $101.5 \pm 31.1$ & 86.4 \\
\hline Nissen SE & ILLUSTRATE & Atorvastatin & 446 & $84.3 \pm 18.9$ & $87.2 \pm 22.6$ \\
\hline Nissen SE & ILLUSTRATE & Atorva+torcetrapib & 464 & $83.1 \pm 19.7$ & $70.1 \pm 25.4$ \\
\hline Kawasaki M & & Control & 17 & $152 \pm 20$ & $149 \pm 24$ \\
\hline Kawasaki M & & Pravastatin & 18 & $149 \pm 19$ & $102 \pm 13$ \\
\hline Kawasaki M & & Atorvastatin & 17 & $155 \pm 22$ & $95 \pm 15$ \\
\hline Hiro T & JAPAN-ACS & Pitavastatin & 125 & $130.9 \pm 33.3$ & $81.1 \pm 23.4$ \\
\hline Hiro T & JAPAN-ACS & Atorvastatin & 127 & $133.8 \pm 31.4$ & $84.1 \pm 27.4$ \\
\hline Nissen SE & ASTEROID & Rosuvastatin & 349 & $130.4 \pm 34.3$ & $60.8 \pm 20.0$ \\
\hline Takayama T & COSMOS & Rosuvastatin & 126 & $140.2 \pm 31.5$ & $82.9 \pm 18.7$ \\
\hline Lee CW & ARTMAP & Atorvastatin & 143 & $110 \pm 31$ & $56 \pm 18$ \\
\hline Lee CW & ARTMAP & Rosuvastatin & 128 & $109 \pm 31$ & $53 \pm 18$ \\
\hline Yamada T & REACH & Atorvastatin & 26 & $123 \pm 17$ & $83 \pm 22$ \\
\hline Yamada T & REACH & Control & 32 & $115 \pm 14$ & $115 \pm 30$ \\
\hline Nasu K & & Fluvastatin & 40 & $144.9 \pm 31.5$ & $98.1 \pm 12.7$ \\
\hline Nasu K & & Control & 39 & $122.3 \pm 18.9$ & $121.0 \pm 21.2$ \\
\hline Nicholls SJ & SATURN & Atorvastatin & 519 & $119.9 \pm 28.9$ & $70.2 \pm 1.0$ \\
\hline Nicholls SJ & SATURN & Rosuvastatin & 520 & $120.0 \pm 27.3$ & $62.6 \pm 1.0$ \\
\hline Hong MK & & Simvastatin & 50 & $119 \pm 30$ & $78 \pm 20$ \\
\hline Hong MK & & Rosuvastatin & 50 & $116 \pm 28$ & $64 \pm 21$ \\
\hline Tani S & & Pravastatin & 52 & $130 \pm 38$ & $104 \pm 20$ \\
\hline Tani S & & Control & 23 & $123 \pm 28$ & $120 \pm 30$ \\
\hline Rodés-C Bef & ERASE & Statins before ACS & 38 & $71 \pm 23$ & $77 \pm 25$ \\
\hline Rodés-C Aft & ERASE & Statins after ACS & 36 & $100 \pm 30$ & $63 \pm 17$ \\
\hline Jensen LO & & Simvastatin & 40 & $158.7 \pm 30.6$ & $85.1 \pm 22.1$ \\
\hline Nissen SE & PERISCOPE & Statins+Gli & 181 & $94.4 \pm 32.9$ & $96.1 \pm 30.4$ \\
\hline Nissen SE & PERISCOPE & Statins+Pio & 179 & $93.5 \pm 30.7$ & $95.6 \pm 28.9$ \\
\hline Nissen SE & STRADIVARIUS & Statins+Rim & 335 & $91.9 \pm 27.9$ & $87.6 \pm 30.5$ \\
\hline Nissen SE & STRADIVARIUS & Statins+Con & 341 & $89.5 \pm 32.2$ & $86.3 \pm 30.3$ \\
\hline
\end{tabular}

Note: *calculated on the bases of baseline levels and change percentage at follow up [21].

\# calculated according to Figure 2 in the paper [15]. 


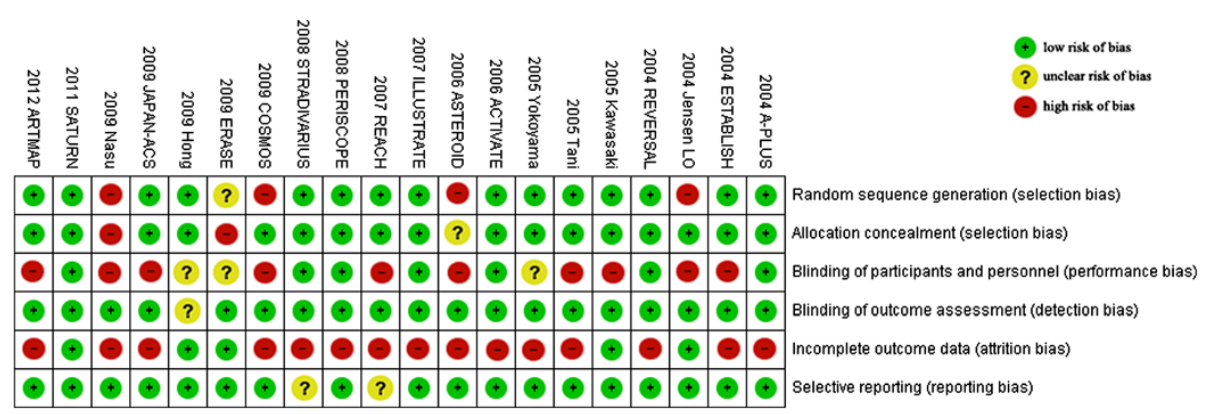

Figure 2 Methodological quality summary of each included trial.

to $-0.214 \mathrm{~mm}^{3}$ (SMD, 95\% CI $-0.342 \sim-0.085$ ) when 2009 COSMOS Ros was omitted; from $-0.093 \mathrm{~mm}^{3}$ (95\% CI -0.174 -0.011) when the arm of 2011 SATURN Ros was omitted to $-0.126 \mathrm{~mm}^{3}$ (SMD, 95\% CI $-0.200 \sim-0.053)$ when 2004 REVERSAL Ato was omitted respectively. Publication bias analysis suggested the values of $p$ by Egger's test were 0.024, 0.605 for group $\geq 30<40$, and group $\geq 40<50$ respectively.

In group $<0$ with mean 19.6 months of follow up and group $\geq 0<30 \%$ with mean 18.3 months of follow up, the volume of CAP at follow up was not significantly decreased, compared with the volumes at baseline (SMD $-0.034 \mathrm{~mm}^{3}, 95 \%$ CI $-0.111 \sim 0.044, p=0.396$; SMD $-0.032 \mathrm{~mm}^{3}, 95 \%$ CI $-0.093 \sim 0.030, p=0.315$ respectively). There was no significant heterogeneity among arms $\left(\chi^{2}\right.$ for heterogeneity $=1.55, p=0.981, I^{2}=0 \%$ for group $<0 \%$; $\chi^{2}$ for heterogeneity $=4.59, p=0.970, I^{2}=0 \%$ for group $\geq 0<30 \%$ ).

Sensitivity analyses showed that LDL-C lowering in group $\geq 0<30 \%$ could not still significantly decrease the volume of CAP with reduction of the CAP volume ranged from $-0.010 \mathrm{~mm}^{3}$ (SMD, 95\% CI: $-0.080 \sim 0.061$ ) when the arm of 2007 ILLUSTRATE Ato + Tor was omitted to $-0.042 \mathrm{~mm}^{3}$ (SMD, 95\% CI: $-0.108 \sim 0.024$ ) when the arm of 2004 REVERSAL Pro was omitted. No publication bias was found, the values of $p$ by Egger's test for group $\geq 0<30 \%$ were 0.537 .
Mean levels of LDL-C at baseline and follow up, mean reducing percentage of LDL-C in group $<0, \geq 0<30, \geq 30<$ $40, \geq 40<50$ and $\geq 50 \%$, were showed in Table 4 .

The effect of lowering LDL-C by statins on regression of coronary atherosclerotic plaque

LDL-C lowering by rosuvastatin, atorvastatin and pitavastatin in group $\leq 70$ and $>70 \leq 100 \mathrm{HP} \mathrm{mg} / \mathrm{dL}$ could lead to regression of CAP, but LDL-C lowering by simvastatin, fluvastatin and pravastatin could not (Figure 6, Table 5).

LDL-C lowering by rosuvastatin (mean $33.3 \mathrm{mg}$ daily for mean 20 months), atorvastatin (mean $60.3 \mathrm{mg}$ daily for mean 17 months) and pitavastatin (4 mg daily for 8 12 months) in group $\leq 70$ and $>70 \leq 100 \mathrm{HP} \mathrm{mg} / \mathrm{dL}$ could significantly decrease the volumes of CAP at follow up, compared with the volumes at baseline (SMD $-0.162 \mathrm{~mm}^{3}$, 95\% CI: $-0.234 \sim-0.081, p=0.000$; SMD $-0.101,95 \%$ CI: $-0.184 \sim-0.019, p=0.016$; SMD $-0.304 \mathrm{~mm}^{3}, 95 \%$ CI: $-0.553 \sim-0.055, p=0.017$; respectively). There was no significant heterogeneity among arms ( $\chi^{2}$ for heterogeneity $=0.37, p=0.985, I^{2}=0 \%$ for rosuvastatin; $\chi^{2}$ for heterogeneity $=4.44, p=0.728, I^{2}=0 \%$ for atorvastatin.

Sensitivity analyses suggested that lowering LDL-C by rosuvastatin could lead to regression of CAP with reduction of the plaque volume ranged from $-0.153 \mathrm{~mm}^{3}$ (SMD, 95\% CI: $-0.249 \sim-0.056$ ) when the arm of 2006 ASTEROID Ros was omitted to $-0.178 \mathrm{~mm}^{3}$ (SMD, 95\%

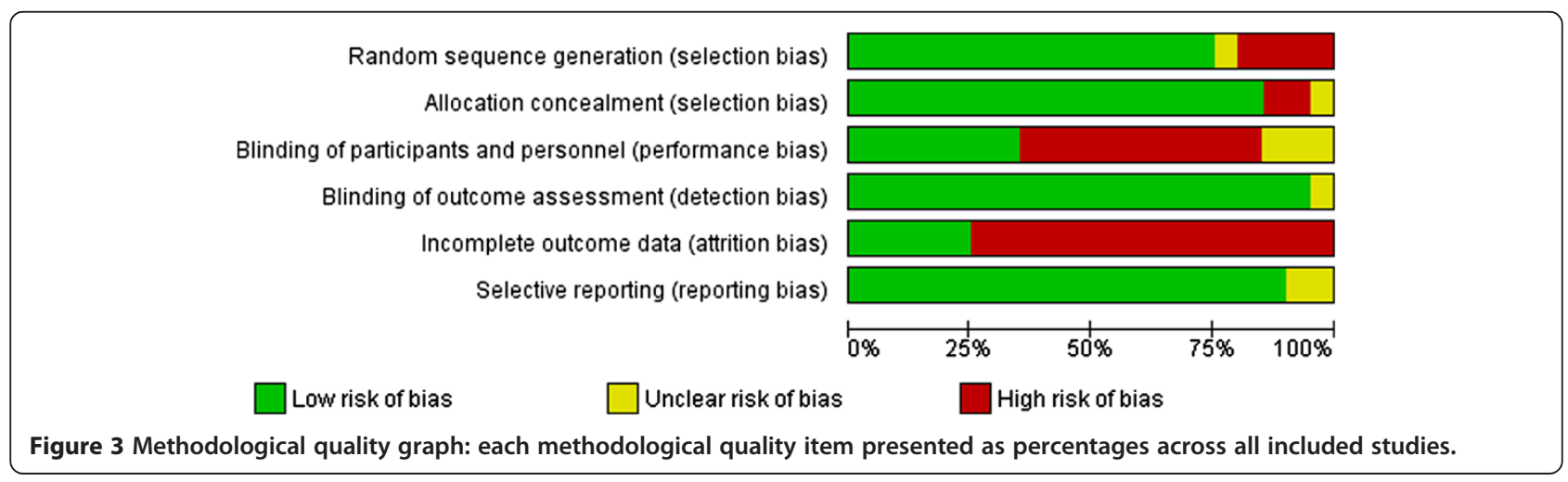




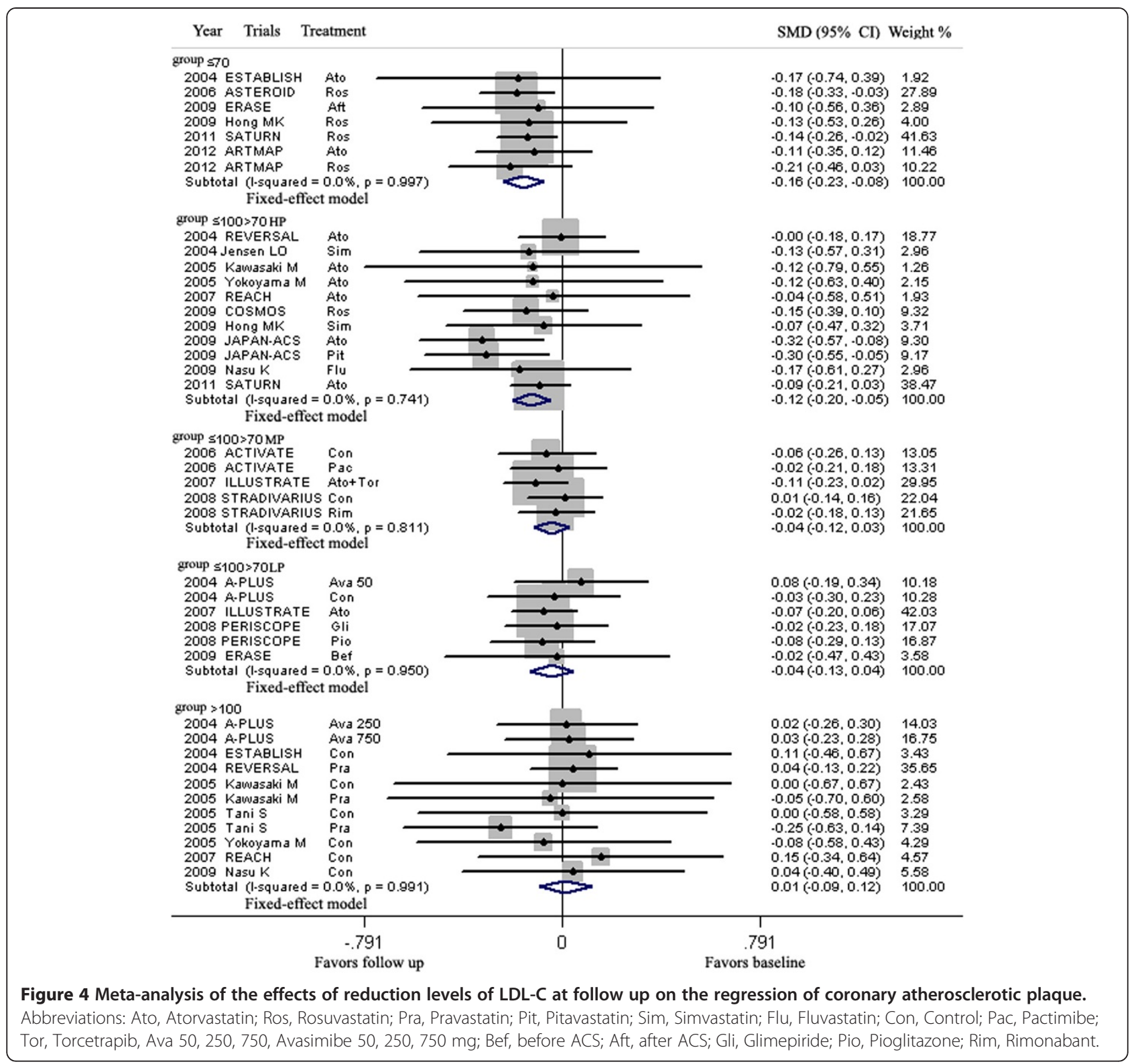

CI: $-0.287 \sim-0.069)$ when the arm of 2011 SATURN Ros was omitted. Lowering LDL-C by atorvastatin could, but not significantly, lead to regression of CAP when the arm of 2009 JAPAN-ACS Ato was omitted (SMD: $-0.075 \mathrm{~mm}^{3}$, 95\% CI: $-0.162 \sim 0.012$ ). No publication bias was found, the values of $p$ by Egger's test for rosuvastatin and atorvastatin group were $0.770,0.582$ respectively (Table 5 ).

Intensity of lowering LDL-C by different statins was shown in Table 6. Rosuvastatin and atorvastatin could reduce LDL-C by more than $40 \%$.

\section{Discussion}

\section{Feature of this meta-analysis}

This meta-analysis broke though the limit of single trial, and pooled arms together according to the levels of
LDL-C at follow up in the arms, regardless of the measures of lowering LDL-C: treating arm (statins, ACAT inhibitor, CETP inhibitor, decreasing obesity drug, and glucose-lowering agents) and control arms (dietary restriction, moderate LDL-C lowering by statin); intensive and moderate LDL-C lowering. The volumes of CAP at follow up were compared with those at baseline in the same arms to evaluate the regression of the CAPs, this meta-analysis really reflected the change of the plaques volume with the change of LDL-C levels.

Our meta-analysis results indicated that intensive lowering LDL-C in group $\leq 70,>70 \leq 100 \mathrm{HP} \mathrm{mg} / \mathrm{dL}$ (mean follow up LDL-C, mean duration of follow up: $60.6 \mathrm{mg} / \mathrm{dL}$, 18.6 months; $77.8 \mathrm{mg} / \mathrm{dL}, 17.4$ months respectively), $\geq 30<$ $40, \geq 40<50$ and $\geq 50 \%$ (mean LDL-C reducing, mean 
Table 3 Results of meta-analysis in each group and mean CAP volume in each group at baseline and follow up

\begin{tabular}{|c|c|c|c|c|c|c|c|c|c|}
\hline \multirow{2}{*}{ Group } & \multirow{2}{*}{$\begin{array}{l}\text { Included } \\
\text { arms (case) }\end{array}$} & \multirow{2}{*}{$\begin{array}{l}\text { CAP Volume at } \\
\text { Baseline }\left(\mathrm{mm}^{3}\right)\end{array}$} & \multirow{2}{*}{$\begin{array}{l}\text { CAP Volume at } \\
\text { Follow up }\left(\mathrm{mm}^{3}\right)\end{array}$} & \multirow{2}{*}{$\begin{array}{l}\text { Pooled SMD } \\
(95 \% \mathrm{Cl}, p)\end{array}$} & \multicolumn{2}{|c|}{$\begin{array}{l}\text { Heterogeneity } \\
\text { test }\end{array}$} & \multicolumn{2}{|c|}{ Sensitivity analyses } & \multirow{2}{*}{$\begin{array}{l}\text { Egger's } \\
\text { test }\end{array}$} \\
\hline & & & & & $\mathrm{X}^{2}$ test $(p)$ & $P^{2}$ & Lower SMD (95\% Cl) & Upper SMD $(95 \% \mathrm{Cl})$ & \\
\hline \multirow{2}{*}{$\leq 70 \mathrm{mg}$} & \multirow{2}{*}{$7(1250)$} & \multirow{2}{*}{$177.1 \pm 41.9$} & \multirow{2}{*}{$125.9 \pm 38.6$} & \multirow{2}{*}{$-0.156(-0.235 \sim-0.078,0.000)$} & \multirow{2}{*}{$0.57(0.997)$} & \multirow{2}{*}{0} & $-0.146(-0.238 \sim-0.054)$ & $-0.167(-0.270 \sim-0.064)$ & \multirow{2}{*}{0.835} \\
\hline & & & & & & & Without 2006 ASTEROID Ros & Without 2011 SATURN Ros & \\
\hline \multirow{2}{*}{$>70 \leq 100 \mathrm{HP} \mathrm{mg}$} & \multirow{2}{*}{$11(1352)$} & \multirow{2}{*}{$129.7 \pm 72.3$} & \multirow{2}{*}{$123.8 \pm 69.8$} & \multirow{2}{*}{$-0.123(-0.199 \sim-0.048,0.001)$} & \multirow{2}{*}{$6.83(0.741)$} & \multirow{2}{*}{0} & $-0.103(-0.182 \sim-0.024)$ & $-0.151(-0.235 \sim-0.067)$ & \multirow{2}{*}{0.501} \\
\hline & & & & & & & Without 2009 JAPAN-ACS Ato & Without 2004 REVERSAL Ato & \\
\hline \multirow{2}{*}{$>70 \leq 100 \mathrm{MP} \mathrm{mg}$} & \multirow{2}{*}{$5(1548)$} & \multirow{2}{*}{$195.8 \pm 2.3$} & \multirow{2}{*}{$191.8 \pm 4.7$} & \multirow{2}{*}{$-0.045(-0.115 \sim-0.026,0.215)$} & \multirow{2}{*}{$1.59(0.811)$} & \multirow{2}{*}{0} & $-0.016(-0.103 \sim-0.066)$ & $-0.061(-0.140 \sim-0.019)$ & \multirow{2}{*}{0.500} \\
\hline & & & & & & & Without 2007 ILLUSTRATE Ato+Tor & Without 2008 STRADIVARIUS Con & \\
\hline \multirow{2}{*}{$>70 \leq 100 L P$ mg } & \multirow{2}{*}{$6(1061)$} & $2012+151$ & $1973+150$ & $-0045(-0130 \sim 00400301)$ & $1.14(0950)$ & 0 & $-0.024(-0.136 \sim 0.087)$ & $-0.059(-0.148 \sim 0.031)$ & 0241 \\
\hline & & $201 . \angle 1+15.1$ & 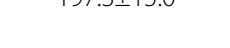 & $=0.045(-0.150 .0 .040,0.501)$ & $1.14(0.950)$ & ( & Without 2007 ILLUSTRATE Ato & Without 2004 A-PLUS Ava 50 & 0.241 \\
\hline$>100 \mathrm{mg}$ & $10(669)$ & $1759+864$ & $1787+891$ & $0.017(-0.090 \sim 01240757)$ & $237(0.984)$ & 0 & $-0.000(-0.135 \sim 0.136)$ & $0.039(-0.073 \sim 0.151)$ & 0692 \\
\hline & & & & & & & Without 2004 REVERSAL Pro & Without 2005 Tani S Pra & \\
\hline$<0 \%$ & $8(1276)$ & $2012+138$ & $1983+138$ & -0034(-0111 0044 0306) & $155(0981)$ & 0 & $-0.012(-0.109 \sim 0.084)$ & $-0.044(-0.125 \sim 0.037)$ & 0087 \\
\hline$-0 \%$ & $0(1270)$ & 201.2113 .0 & 年 & 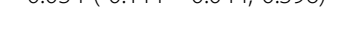 & (1.0 & ( & Without 2007 ILLUSTRATE Ato & Without 2004 A-PLUS Ava 50 & \\
\hline$>0<30 \%$ & $13(2014)$ & $1886+517$ & $1863+527$ & $-0032(-0093 \sim 00300315)$ & 450 (0970) & 0 & $-0.010(-0.080 \sim 0.061)$ & $-0.042(-0.108 \sim 0.024)$ & 0537 \\
\hline$>0 \leq 30 \%$ & $13(2014)$ & $188.0 \pm 31.1$ & $180.3 \pm 32.1$ & $-0.032(-0.093 \sim 0.030,0.313)$ & $4.59(0.9 / 0)$ & 0 & Without 2007 ILLUSTRATE Ato+Tor & Without 2004 REVERSAL Pra & 0.531 \\
\hline$>30 \leq 40 \%$ & $10(594)$ & $102.9+969$ & $94.3+90.4$ & $-0.199(-0.314 \sim-0.085,0.001)$ & $3.10(0.960)$ & 0 & $-0.166(-0.295 \sim-0.038)$ & $-0.214(-0.342 \sim-0.085)$ & 2024 \\
\hline & & & & & & & Without 2009 JAPAN-ACS Ato & Without 2009 COSMOS Ros & $0.0 \angle 4$ \\
\hline$>40<50 \%$ & $8(1677)$ & $1578+378$ & $1507+363$ & - 1088 (-0176 - -0040 (2) & 250 (0997) & 0 & $-0.093(-0.174 \sim-0.011)$ & $-0.126(-0.200 \sim-0.053)$ & 0605 \\
\hline 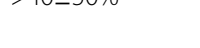 & (1) & 年 & 年 & 0.100 ( $0.170 .0 .070,0.002)$ & $2.00(0.021)$ & 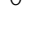 & Without 2011 SATURN Ros & Without 2004 REVERSAL Ato & 0.000 \\
\hline$>50 \%$ & 1 (349) & $212.2 \pm 81.3$ & $197.5 \pm 79.1$ & $-0.183(-0.332 \sim-0.035,0.016)$ & & & & & \\
\hline
\end{tabular}


Table 4 Levels and reducing percentage of LDL-C and duration in each group

\begin{tabular}{|c|c|c|c|c|c|c|}
\hline Group & $\mathbf{N}$ & $\begin{array}{l}\text { Mean LDL-C at } \\
\text { Baseline (mg) }\end{array}$ & $\begin{array}{l}\text { Mean LDL-C at } \\
\text { Follow up (mg) }\end{array}$ & $\begin{array}{l}\text { Mean Reducing } \\
\text { percentage }\end{array}$ & $\begin{array}{l}\text { Actual range of } \\
\text { reducing percentage }\end{array}$ & Duration (month) \\
\hline$\leq 70 \mathrm{mg}$ & 1250 & $120.0 \pm 8.2$ & $60.6 \pm 3.5$ & $48.8 \pm 3.3$ & $37 \sim 53.2$ & $18.6 \pm 8.2$ \\
\hline$>70 \leq 100 \mathrm{HP} \mathrm{mg}$ & 1352 & $132.4 \pm 12.9$ & $77.8 \pm 7.0$ & $40.4 \pm 4.0$ & $32.3 \sim 46.7$ & $17.4 \pm 5.9$ \\
\hline$>70 \leq 100 \mathrm{MP} \mathrm{mg}$ & 1548 & $91.3 \pm 6.9$ & $82.4 \pm 8.2$ & $9.1 \pm 4.5$ & $3.6 \sim 14.9$ & $19.8 \pm 2.7$ \\
\hline$>70 \leq 100 \mathrm{LP} \mathrm{mg}$ & 1061 & $88.5 \pm 5.5$ & $91.5 \pm 5.4$ & $-4.7 \pm 2.5$ & $-1.7 \sim-8.5$ & $19.9 \pm 4.5$ \\
\hline$>100 \mathrm{mg}$ & 699 & $125.1 \pm 24.4$ & $110.0 \pm 9.3$ & $8.3 \pm 15.6$ & $-10.9 \sim 32$ & $14.6 \pm 5.1$ \\
\hline$<0 \%$ & 1276 & $89.1 \pm 5.3$ & $93.2 \pm 6.2$ & $-5.6 \pm 3.1$ & $-1.7 \sim-10.9$ & $19.6 \pm 4.2$ \\
\hline$>0 \leq 30 \%$ & 2014 & $102.4 \pm 22.1$ & $89.7 \pm 15.7$ & $10.6 \pm 7.3$ & $0 \sim 25$ & $18.3 \pm 4.5$ \\
\hline$>30 \leq 40 \%$ & 594 & $132.6 \pm 11.4$ & $83.3 \pm 7.7$ & $36.1 \pm 1.9$ & $32 \sim 39$ & $10.3 \pm 3.1$ \\
\hline$>40 \leq 50 \%$ & 1677 & $123.7 \pm 13.4$ & $66.8 \pm 8.0$ & $45.4 \pm 2.8$ & $41.5 \sim 49$ & $19.4 \pm 6.9$ \\
\hline$>50 \%$ & 349 & $130.4 \pm 34.3$ & $60.8 \pm 20.0$ & 53.2 & 53.2 & 24 \\
\hline
\end{tabular}

duration of follow up: $36.1 \%, 10.3$ months; $45.4 \%$, 19.4 months; $53.2 \%$, 24 months respectively) could lead to the regression of CAP; that moderate lowering LDL-C in group $>70 \leq 100 \mathrm{MP} \mathrm{mg} / \mathrm{dL}$ (mean $\mathrm{LDL}-\mathrm{C}$ reducing by 9.1\%, mean 19.8 months of follow up), $>100$ (mean follow up LDL-C 110.0 and mean 14.6 months of follow up) $\mathrm{mg} / \mathrm{dL}$ and $\geq 0<30 \%$ (mean LDL-C reducing by $10.6 \%$, mean 18.3 months of follow up) could not lead to the regression; and that intensive lowering LDL-C, by mean $48 \%$ with rosuvastatin, and by mean $42 \%$ with atorvastatin, could regress CAP. The sensitivity analysis confirmed the effect of the LDL-C change on the volume of the plaque.

\section{The importance of intensive lowing LDL-C on regression of CAP and LDL-C target of this meta-analysis}

In the trials that evaluated the effects of LDL-C lowering on atheroma progression by IVUS, the effects varied with level of LDL-C at follow up. In group $\leq 70 \mathrm{mg}, \geq 30<40 \%$ and $\geq 40<50 \%$, the LDL-C at baseline in most trials (including ESTABLISH [14], REVERSAL [13], JAPAN-ACS [12], ASTEROID [37], COSMOS [40], trial by Kawasaki $\mathrm{M}$ [16] and by Nasu K [41]) were $>120 \mathrm{mg}$. In ASTEROID [37], COSMOS [40], JAPAN-ACS [12] trial and fluvastatin arm of the trial by Nasu $\mathrm{K}$ [41] with respective the mean LDL-C level $60.8 \mathrm{mg}, 82.9 \mathrm{mg}, 81-84 \mathrm{mg}$ and $98 \mathrm{mg}$ (53.2\%, 38.6\%, 36\% and 32.3\% reduction of level of LDL-C) at follow up, it was showed that CAP could be regressed with intensive statin therapy. In ESTABLISH [14] and REVERSAL [13], the mean reducing percent of LDL-C at follow up in the statin treatment arms were $44 \%$ and $46 \%$ respectively, the volumes of CAPs at follow up were not significantly decreased, compared with those in baseline. In the trails by Yokoyama $M$ [15] and Kawasaki M [16], mean reducing percentage of LDL-C at follow up was 35\% for atorvastatin arm of the trial by Yokoyama $M$ [15], 32\% for pravastatin arm of the trial by Kawasaki M [16] and 39\% for atorvastatin arm of the trial by Kawasaki M [16], the volume of CAPs at follow up were also not significantly decreased, compared with that at baseline. Pooled these arms with follow up LDL-C $\leq 70 \mathrm{mg}$ or reducing $>30 \%$ together, these meta-analysis showed that the CAPs could be regressed in group $\leq 70 \mathrm{mg}, \geq 30<40 \%$ and $\geq 40<50 \%$. Because of publication bias in group $\geq 30<40 \%$ (Table 3 ), the level of LDL-C in this group could not be recommended for regressing CAP. Based on the mean level and reducing percentage of LDL-C in group $\leq 70 \mathrm{mg}$ and $\geq 40<50 \%$ $(60.6 \pm 3.5 \mathrm{mg}, 48.8 \pm 3.3 \% ; 66.8 \pm 8.0 \mathrm{mg}, 45.4 \pm 2.8 \%$, in Table 4), the meta-analysis in group $\leq 70 \mathrm{mg}$ and $\geq 40<$ $50 \%$ suggested that for regressing CAP, LDL-C should be reduced by $>45 \%$ or to a target level $\leq 66 \mathrm{mg} / \mathrm{dL}$.

In trials with 18-24 months of non-statin (ACAT inhibitor, decreasing obesity drugs and glucose-lowering agents) treatment, although the levels of LDL-C at follow up in some arms (ACTIVATE [22], STRADIVARIUS [35], PERISCOPE [36], and A-PLUS [21] with daily $50 \mathrm{mg}$ of avasimibe) were $>70 \leq 100 \mathrm{mg} / \mathrm{dL}$, the LDL-C lowering percentage at follow up in the arms were below $30 \%$ because the levels of LDL-C at baseline were $<95 \mathrm{mg} / \mathrm{dL}$. In ILLUSTRATE trial [23], after treatment with atorvastatin to reduce levels of LDL-C to less than $100 \mathrm{mg} / \mathrm{dL}$, patients were randomly assigned to receive either atorvastatin monotherapy or atorvastatin plus $60 \mathrm{mg}$ of torcetrapib daily. After 24 months, the reduction of LDL-C in both arms was $<24 \%$ and the progression of CAP was not halted. In trial $[34,35]$ with statins treatment and baseline LDL-C $<110 \mathrm{mg}$, if the LDL-C lowering percentage at follow up were $<24 \%$, the CAP was also not regressed. The meta analysis with six arms in group $>70 \leq 100 \mathrm{LP}$ $\mathrm{mg} / \mathrm{dL}$ and five arms in group $>70 \leq 100 \mathrm{MP} \mathrm{mg} / \mathrm{dL}$ did not show that only $>70 \leq 100 \mathrm{mg} / \mathrm{dL}$ of LDL-C level but $<30 \%$ reduction at follow up could lead to regression of CAP, which further confirmed the importance of intensively lowering LDL-C in regression of CAP. Though LDL-C at follow up in some trials $[13,15,16,26,27,39]$ of LDL-C lowering by statins was $>70 \leq 100 \mathrm{mg} / \mathrm{dL}$ and 


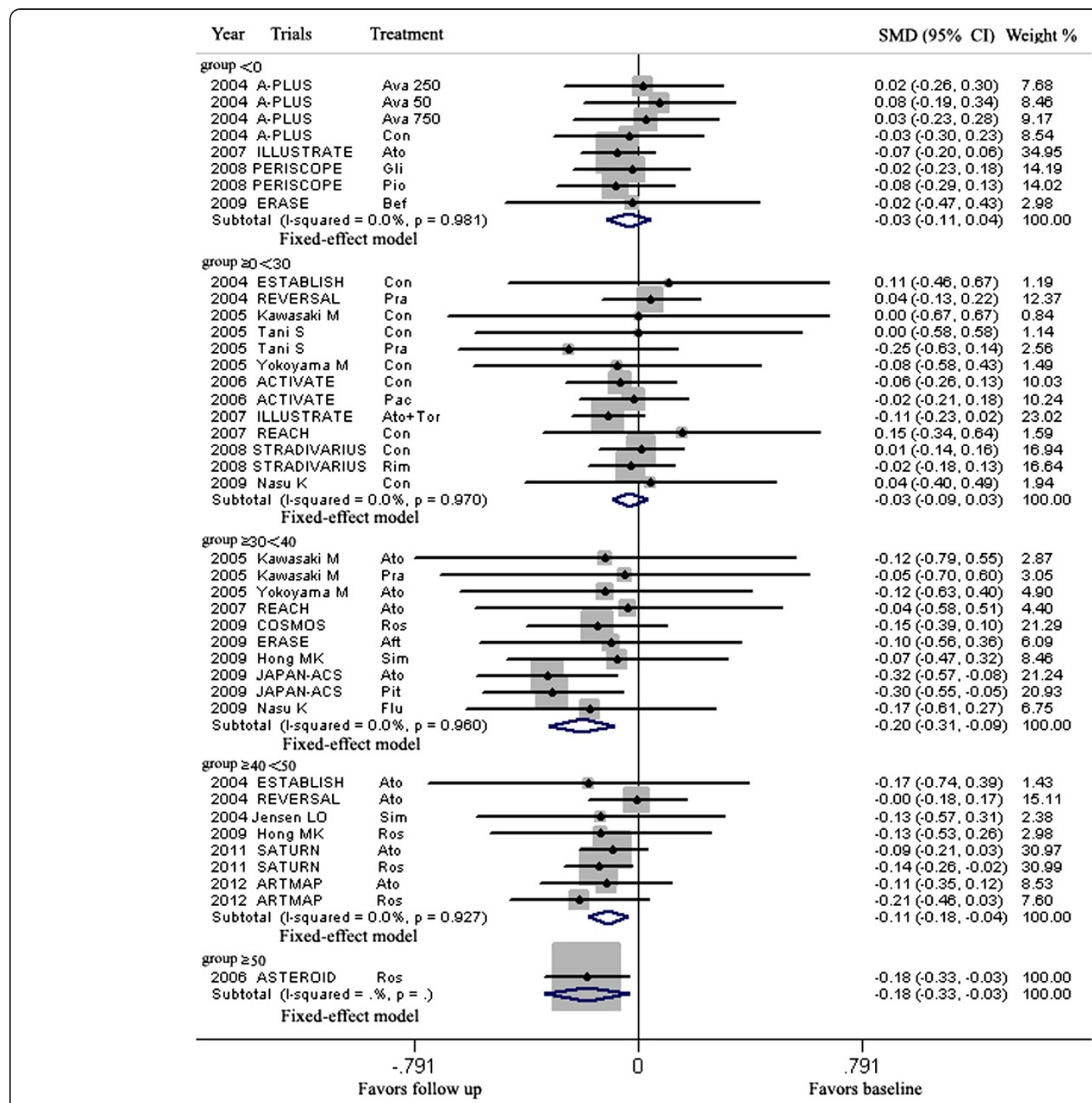

Figure 5 Meta-analysis of the effects of reduction percentages of LDL-C at follow up on the regression of coronary atherosclerotic plaque. Abbreviations: Ato, Atorvastatin; Ros, Rosuvastatin; Pra, Pravastatin; Pit, Pitavastatin; Sim, Simvastatin; Flu, Fluvastatin; Con, Control; Pac, Pactimibe; Tor, Torcetrapib, Ava 50, 250, 750, Avasimibe 50, 250, 750 mg; Bef, before ACS; Aft, after ACS; Gli, Glimepiride; Pio, Pioglitazone; Rim, Rimonabant.

reducing $>30 \%$, the CAP in the trials was also not regressed. Included eleven arms with baseline LDL-C > $130.0 \mathrm{mg} / \mathrm{dL}$, follow up LDL-C $>70 \leq 100 \mathrm{mg} / \mathrm{dL}$ and LDL-C reducing $>30 \%$ (in group $>70 \leq 100 \mathrm{HP} \mathrm{mg}$ ), this meta-analysis suggested that LDL-C reducing $>40 \%$ or to target $77.8 \mathrm{mg}$ could regress CAP (Table 4). The metaanalysis in group $>70 \leq 100 \mathrm{HP},>70 \leq 100 \mathrm{MP}$ and $>70 \leq$ $100 \mathrm{LP} \mathrm{mg} / \mathrm{dL}$ indicated that LDL-C reducing percentage, not lowering absolute value of LDL-C at follow up, was important for regressing CAP.

Although rosuvastatin, atorvastatin, pitavastatin, simvastatin, and fluvastatin in some trials could reduce LDL-C level to $\leq 100 \mathrm{mg}$ or by $30 \%$, the meta-analysis indicated that rosuvastatin, atorvastatin and pitavastatin (mean lowering LDL-C by $48.4 \%, 42.3 \%$ and $36.2 \%$ respectively) could regress the CAPs, and simvastatin with mean lowering LDL-C by $39.9 \%$ could not. The role of pitavastatin in regressing CAPs remains to be verified because the role was from only one RCT with 125 cases [12]. Pravastatin with mean lowering LDL-C by $24.6 \%$ could not regress the CAPs either. Fluvastatin with mean lowering LDL-C by $32.3 \%$ in the blinded endpoint trial with 40 patients can regress the CAP [41], but meta-analysis indicated that fluvastatin could not regress the CAP. The reason that pravastatin and fluvastatin in this meta-analysis can not regress the CAPs might be attributed to their low-intensity of lowering LDL$\mathrm{C}$ and low dosage which can not reduce LDL-C by $>40 \%$.

Taken all the results of meta-analysis together, it was recommended that LDL-C level should be reduced by $>40 \%$ or to a target level $<78 \mathrm{mg} / \mathrm{dL}$ for regressing CAP. 


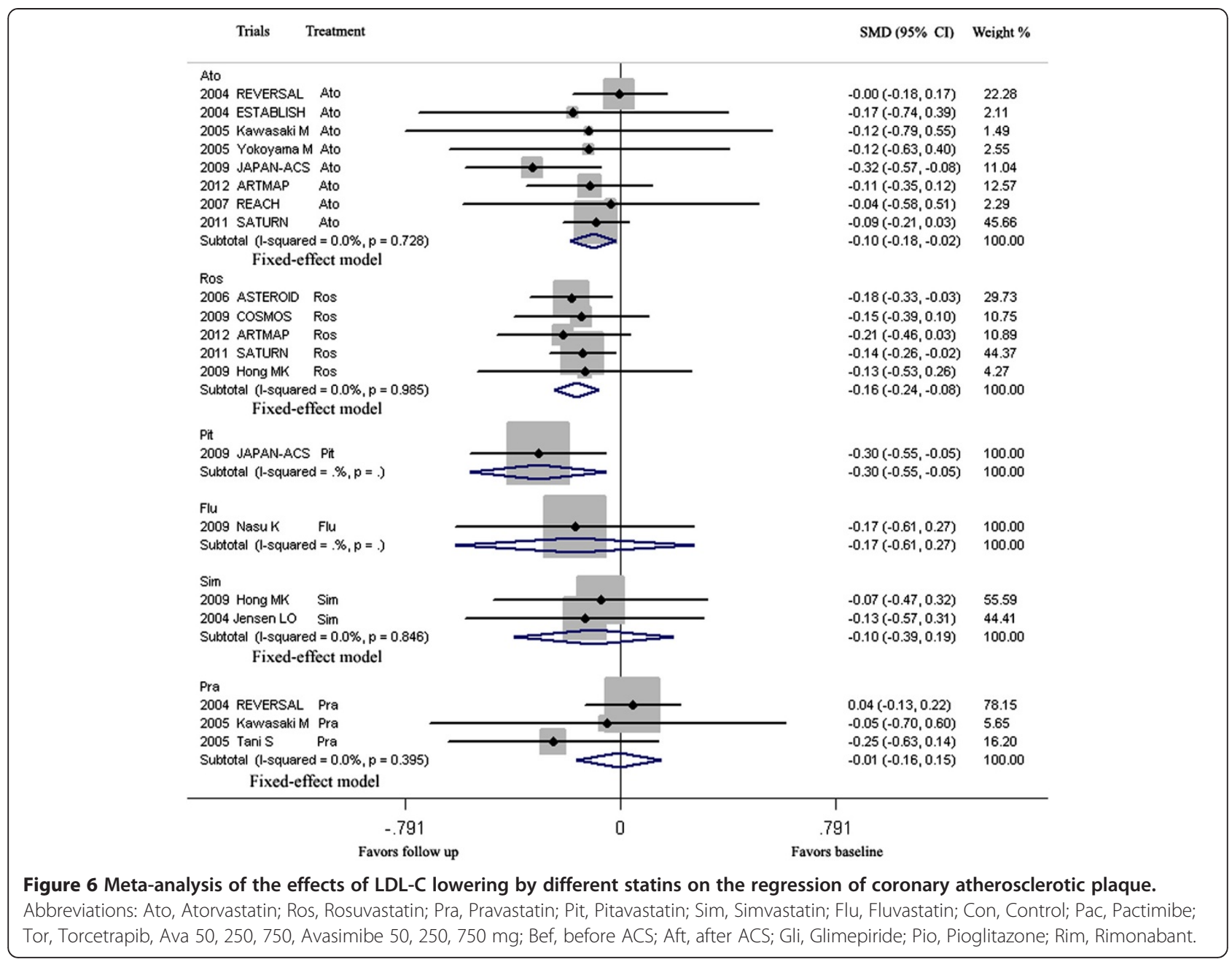

The difference of LDL-C target level between this meta-analysis and current guidelines

The patients included in this meta-analysis were coronary heart disease. According to 2004 the guideline of the Adult Treatment Panel III (ATP III) of the National
Cholesterol Education Program [43] and 2011 ESC/EAS Guidelines for the management of dyslipidaemias [1], this group of patients belongs to very high risk category, and the recommended targets of LDL-C should be less than $70 \mathrm{mg} / \mathrm{dL}$ or $30-40 \%$ reduction from baseline in

Table 5 Results of meta-analysis in different statins groups

\begin{tabular}{|c|c|c|c|c|c|c|c|}
\hline \multirow[t]{2}{*}{ Group } & \multirow{2}{*}{$\begin{array}{l}\text { Included arms } \\
\text { (and case) }\end{array}$} & \multirow{2}{*}{ Pooled SMD (95\% Cl, p) } & \multicolumn{2}{|c|}{$\begin{array}{l}\text { Heterogeneity } \\
\text { test }\end{array}$} & \multicolumn{2}{|c|}{ Sensitivity analyses } & \multirow{2}{*}{$\begin{array}{c}\text { Egger's } \\
\text { test }\end{array}$} \\
\hline & & & $x^{2}$ test $(p)$ & $1^{2}$ & Lower SMD (95\% Cl) & Upper SMD (95\% CI) & \\
\hline \multirow{2}{*}{ Rosuvastatin } & \multirow{2}{*}{$5(1173)$} & \multirow{2}{*}{$-0.162(-0.234 \sim-0.081,0.000)$} & \multirow{2}{*}{$0.37(0.985)$} & \multirow{2}{*}{0} & $-0.153(-0.249 \sim-0.056)$ & $-0.178(-0.287 \sim-0.069)$ & \multirow{2}{*}{0.770} \\
\hline & & & & & Without 2006 ASTEROID Ros & Without 2011 SATURN Ros & \\
\hline \multirow{2}{*}{ Atorvastatin } & \multirow{2}{*}{$8(1138)$} & \multirow{2}{*}{$-0.101(-0.184 \sim-0.019,0.016)$} & \multirow{2}{*}{$4.44(0.728)$} & \multirow{2}{*}{0} & $-0.075(-0.162 \sim 0.012)$ & $-0.132(-0.225 \sim-0.038)$ & \multirow{2}{*}{0.582} \\
\hline & & & & & Without 2009 JAPAN-ACS Ato & Without 2004 REVERSAL Ato & \\
\hline Pitavastatin & $1(125)$ & $-0.304(-0.553 \sim-0.055,0.017)$ & & & & & \\
\hline Fluvastatin & $1(40)$ & $-0.169(-0.608 \sim 0.270,0.450)$ & & & & & \\
\hline \multirow{2}{*}{ Simvastatin } & \multirow{2}{*}{$2(90)$} & \multirow{2}{*}{$-0.10(-0.393 \sim 0.192,0.501)$} & \multirow{2}{*}{$0.04(0.846)$} & \multirow{2}{*}{0} & $-0.074(-0.467 \sim 0.318)$ & $-0.133(-0.572 \sim 0.360)$ & \multirow{2}{*}{0.000} \\
\hline & & & & & Without 2004 Jensen LO Sim & Without 2009 Hong MK Sim & \\
\hline \multirow{2}{*}{ Pravastatin } & \multirow{2}{*}{$3(319)$} & \multirow{2}{*}{$-0.008(-0.163 \sim 0.147,0.920)$} & \multirow{2}{*}{$1.86(0.395)$} & \multirow{2}{*}{0} & $-0.005(-0.165 \sim 0.154)$ & $0.039(-0.131 \sim 0.208)$ & \multirow{2}{*}{0.528} \\
\hline & & & & & Without 2005 Kawasaki M Pra & Without 2005 Tani S Pra & \\
\hline
\end{tabular}


Table 6 Levels and reducing percentage of LDL-C, dosage and duration in different statin group

\begin{tabular}{|c|c|c|c|c|c|c|c|}
\hline Group & $\mathrm{N}$ & Age & $\begin{array}{c}\text { MeanLDL-C } \\
\text { at Baseline }(\mathrm{mg})\end{array}$ & $\begin{array}{l}\text { MeanLDL-C at } \\
\text { Follow up (mg) }\end{array}$ & $\begin{array}{c}\text { Mean Reducing } \\
\text { percentage }\end{array}$ & Statin dosage $(\mathrm{mg})$ & Duration (month) \\
\hline Rosuvastatin & 1173 & $58.1 \pm 1.8$ & $123.9 \pm 8.6$ & $63.3 \pm 7.4$ & $48.4 \pm 4.2$ & $33.3 \pm 11.6$ & $20.5 \pm 6.3$ \\
\hline Atorvastatin & 1138 & $58.4 \pm 2.5$ & $128.0 \pm 14.0$ & $73.0 \pm 8.7$ & $42.3 \pm 3.7$ & $60.3 \pm 28.6$ & $17.5 \pm 7.1$ \\
\hline Pitavastatin & 125 & $62.5 \pm 11.5$ & $130.9 \pm 33.3$ & $81.1 \pm 23.4$ & $36.2 \pm 19.5$ & 4 & $8 \sim 12$ \\
\hline Fluvastatin & 40 & $63.0 \pm 10.0$ & $144.9 \pm 31.5$ & $98.1 \pm 12.7$ & 32.3 & 60 & 12 \\
\hline Simvastatin & 90 & $57.9 \pm 0.1$ & $136.61 \pm 5.3$ & $81.2 \pm 3.5$ & $39.9 \pm 6.1$ & $28.9 \pm 10.0$ & $17.8 \pm 6.5$ \\
\hline Pravastatin & 319 & $58.2 \pm 3.2$ & $146.8 \pm 7.4$ & $108.9 \pm 2.9$ & $24.6 \pm 2.6$ & $34.8 \pm 9.9$ & $15.4 \pm 5.0$ \\
\hline
\end{tabular}

ATP III, and less than $70 \mathrm{mg} / \mathrm{dL}$ or a $\geq 50 \%$ reduction in 2011 ESC/EAS Guidelines. The target levels for subjects at very high risk in the both guidelines are extrapolated from several clinical trials [43], mainly from the metaanalysis by Cholesterol Treatment Trialists' Collaborators [44], which indicated that absolute benefit of LDL-C lowering related chiefly to the absolute reduction of $\mathrm{LDL}-\mathrm{C}$, and the risk reductions are proportional to the absolute LDL-C reductions, but the meta-analysis did not provide target level of LDL-C for the benefit in terms of cardiovascular disease reduction [44]. According to 2013 ACC/AHA blood cholesterol guideline [45], this group of patients should be treated with highintensity statin (atorvastatin $40-80 \mathrm{mg}$ daily or rosuvastatin 20-40 mg daily), which was the intensity of statin suggested in this meta-analysis (Table 6).

The results of our meta-analysis imply that the patients with CHD should be intensively treated with statins (rosuvastatin $33 \mathrm{mg}$ or atorvastatin $60 \mathrm{mg}$ daily) to reduce the level of LDL-C by $>40 \%$ or to a target level $<78 \mathrm{mg} / \mathrm{dL}$ for regressing CAP, which have a little different to the guidelines. These different targets level of LDL-C might be due to different observational index: cardiovascular events for both guidelines, CAP volume for this meta-analysis. Moreover, our target is directly from meta-analysis, the target of 2011 ESC/EAS Guidelines is from extrapolation of metaanalysis, not a direct data. Our meta-analysis revealed the relation between the regression of coronary artery disease and LDL-C level from the view of pathological anatomy. Published meta-analysis $[17,18]$ about CAP by IVUS did not review the relationship between LDL-C level and CAP.

\section{Study limitation}

The results of this analysis were obtained by pooling data from twenty clinical trials. As with any metaanalysis, this study has some limitations. Firstly, though no publication bias was observed by Egger's test there may be a potential of publication bias because only published data were included. Secondly, the methodology used for measurement of coronary atheroma might not be the same in the studies. The plaques volume may be calculated from slices with $1 \mathrm{~mm}$ apart for a length of $10 \mathrm{~mm}$ vessel in some trials [13,15,22,23,27-29,37], or 0.1-0.3 mm-apart for a length of $10-50 \mathrm{~mm}$ vessel in other trials $[12,21,33,39,40]$, which might affect accuracy of plaque measurement. There were some differences in selecting plaque: some trials assessed the plaque in nonculprit vessel, while others assessed non-culprit plaque in a culprit vessel $[12,14,34]$, which assured the plaque was stable. Our study focus on target plaque change, i.e. plaque regression or progression, those differences in measurements and plaque selection did not affect the change of the target plaque with LDL-C levels. So, it has little effect on homogeneous of studies, and this detection bias was very much limited from values of $P$ in $X^{2}$ test and $I^{2}$ in each group. Thirdly, follow up duration might have some effects of the changes of CAP. Fourthly, other cardiovascular risk factors but LDL-C levels, for example, demographic characteristics such as age, gender and ethnicity, might also affect the effect of LDL-C on CAP, and the effects of these factors on CAP remain to be investigated in future.

\section{Implication for practice}

This meta-analysis investigated the effect of reduction of LDL-C only on the regression of the plaque, not on reduction of cardiovascular events. In fact, all the included trial have no the data about death because only the alive have IVUS data at follow up. But in four-year of the OLIVUS-Ex [46], it was found that patients with annual atheroma progression had more adverse cardio- and cerebrovascular events than the rest of the population. A meta-analysis [47] included 7864 CAD patients showed that rates of plaque volume regression were significantly associated with the incidence of MI or revascularization, and it was concluded that regression of atherosclerotic coronary plaque volume in stable CAD patients may represent a surrogate for myocardial infarction and repeat revascularization. Plaque in $\mathrm{CAD}$, as blood pressure level in hypertension, is not major adverse cardiac events, but does be an important surrogate. Therefore, the conclusion of this meta-analysis not only applies to guide LDL-C lowering therapy for regressing CAP, may also apply to guide LDL-C lowering therapy for reducing 
major adverse cardio- and cerebrovascular events. Furthermore, high level of LDL-C plays a crucial role in the formation of atherosclerotic plaque, but LDL-C level is not unique risk factor for atherosclerotic plaque. Hypertension is another important risk factor for the formation of plaque $[48,49]$. Smoking cessation, administrating $\beta$-blockers, anti-hypertension therapy might play some role in slowing progression of CAP [48,50-52]. The trend of CAP regression in group $<0 \%$ might attribute to these non-LDL-C reducing factors.

\section{Conclusions}

Atherosclerotic plaque extension and disruption are basic mechanism of atherosclerotic cardio- and cerebrovascular disease. Stabling and regressing atherosclerotic plaque play an important role in preventing cardio- and cerebrovascular disease. Pooled the twenty trials with CAP detected by gold standard: IVUS, this systemic review demonstrated that intensive lowering LDL-C (rosuvastatin mean $33 \mathrm{mg}$ daily and atorvastatin mean $60 \mathrm{mg}$ daily) with $>17$ months of duration could lead to the regression of coronary atherosclerotic plaque, LDL-C level should be reduced by $>40 \%$ or to a target level $<78 \mathrm{mg} / \mathrm{dL}$ for regressing CAP.

\section{Abbreviations \\ LDL-C: Low-density lipoprotein cholesterol; CAP: Coronary atherosclerotic plaque; CHD: Coronary heart disease; RCT: Randomized controlled trial; CAG: Coronary angiography; IVUS: Intravascular ultrasound; SMD: Standardized mean differences; ACS: Acute coronary syndrome; ACAT: Acyl-coenzyme A:cholesterol acyltransferase; CETP: Cholesteryl ester transfer protein; Cl: Confidence interval; ATP III: Adult Treatment Panel III; CAD: Coronary artery disease.}

\section{Competing interests}

The authors declare that they have no competing interests. This study was not funded.

\section{Authors' contributions}

GWQ, FQZ and LYF carried out data extraction, participated in the analysis and drafted the manuscript. LYX and LCY participated in the design of the study and helped to draft the manuscript. HY, CYM and YB conceived the study, and participated in its statistical analysis. All authors read and approved the final manuscript.

\section{Author details}

${ }^{1}$ The Department of Cardiology, Chinese PLA General Hospital, Beijing 100853, China. ${ }^{2}$ The First Department of Geriatric Cardiology, Chinese PLA General Hospital, Beijing 100853, China. ${ }^{3}$ Navy Wangshoulu Clinics, Xicui Road, Beijing 100036, China.

Received: 2 January 2014 Accepted: 25 April 2014 Published: 2 May 2014

\section{References}

1. Reiner Z, Catapano AL, De Backer G, Graham I, Taskinen MR, Wiklund O, Agewall S, Alegria E, Chapman MJ, Durrington P, Erdine S, Halcox J, Hobbs R, Kjekshus J, Filardi PP, Riccardi G, Storey RF, Wood D: ESC/EAS Guidelines for the management of dyslipidaemias: the Task Force for the management of dyslipidaemias of the European Society of Cardiology (ESC) and the European Atherosclerosis Society (EAS). Eur Heart J 2011, 32:1769-1818.

2. Falk E, Shah PK, Fuster V: Coronary plaque disruption. Circulation 1995, 92:657-671.
3. The Long-Term Intervention with Pravastatin in Ischaemic Disease (LIPID) Study Group: Prevention of cardiovascular events and death with pravastatin in patients with coronary heart disease and a broad range of initial cholesterol levels. N Engl J Med 1998, 339:1349-1357.

4. Scandinavian Simvastatin Survival Study Group: Randomised trial of cholesterol lowering in $\mathbf{4 4 4 4}$ patients with coronary heart disease: the Scandinavian Simvastatin Survival Study (4S). Lancet 1994, 344:1383-1389.

5. Brown G, Albers JJ, Fisher LD, Schaefer SM, Lin JT, Kaplan C, Zhao XQ, Bisson $B D$, Fitzpatrick VF, Dodge HT: Regression of coronary artery disease as a result of intensive lipid-lowering therapy in men with high levels of apolipoprotein B. N Engl J Med 1990, 323:1289-1298.

6. Blankenhorn DH, Azen SP, Kramsch DM, Mack WJ, Cashin-Hemphill L, Hodis HN, DeBoer LW, Mahrer PR, Masteller MJ, Vailas LI, Alaupovic P, Hirsch LJ: Coronary angiographic changes with lovastatin therapy. The Monitored Atherosclerosis Regression Study (MARS). Ann Intern Med 1993, 119:969-976.

7. Thomas AC, Davies MJ, Dilly S, Dilly N, Franc F: Potential errors in the estimation of coronary arterial stenosis from clinical arteriography with reference to the shape of the coronary arterial lumen. Br Heart J 1986, 55:129-139.

8. Hausmann D, Johnson JA, Sudhir K, Mullen WL, Friedrich G, Fitzgerald PJ, Chou TM, Ports TA, Kane JP, Malloy MJ, Yock PG: Angiographically silent atherosclerosis detected by intravascular ultrasound in patients with familial hypercholesterolemia and familial combined hyperlipidemia: correlation with high density lipoproteins. J Am Coll Cardiol 1996, 27:1562-1570.

9. Mintz GS, Painter JA, Pichard AD, Kent KM, Satler LF, Popma JJ, Chuang YC Bucher TA, Sokolowicz LE, Leon MB: Atherosclerosis in angiographically "normal" coronary artery reference segments: an intravascular ultrasound study with clinical correlations. J Am Coll Cardiol 1995, 25:1479-1485.

10. Nissen SE, Yock P: Intravascular ultrasound: novel pathophysiological insights and current clinical applications. Circulation 2001, 103:604-616.

11. Takagi T, Yoshida K, Akasaka T, Hozumi T, Morioka S, Yoshikawa J: Intravascular ultrasound analysis of reduction in progression of coronary narrowing by treatment with pravastatin. Am J Cardiol 1997, 79:1673-1676.

12. Hiro T, Kimura T, Morimoto T, Miyauchi $K$, Nakagawa $Y$, Yamagishi M, Ozaki Y, Kimura K, Saito S, Yamaguchi T, Daida H, Matsuzaki M: Effect of intensive statin therapy on regression of coronary atherosclerosis in patients with acute coronary syndrome: a multicenter randomized trial evaluated by volumetric intravascular ultrasound using pitavastatin versus atorvastatin (JAPAN-ACS [Japan assessment of pitavastatin and atorvastatin in acute coronary syndrome] study). J Am Coll Cardiol 2009, 54:293-302.

13. Nissen SE, Tuzcu EM, Schoenhagen P, Brown BG, Ganz P, Vogel RA, Crowe T, Howard G, Cooper CJ, Brodie B, Grines CL, DeMaria AN: Effect of intensive compared with moderate lipid-lowering therapy on progression of coronary atherosclerosis: a randomized controlled trial. JAMA 2004, 291:1071-1080.

14. Okazaki S, Yokoyama T, Miyauchi K, Shimada K, Kurata T, Sato H, Daida H: Early statin treatment in patients with acute coronary syndrome: demonstration of the beneficial effect on atherosclerotic lesions by serial volumetric intravascular ultrasound analysis during half a year after coronary event: the ESTABLISH Study. Circulation 2004, 110:1061-1068.

15. Yokoyama M, Komiyama N, Courtney BK, Nakayama T, Namikawa S, Kuriyama N, Koizumi T, Nameki M, Fitzgerald PJ, Komuro I: Plasma low-density lipoprotein reduction and structural effects on coronary atherosclerotic plaques by atorvastatin as clinically assessed with intravascular ultrasound radio-frequency signal analysis: a randomized prospective study. Am Heart $J$ 2005, 150:287.

16. Kawasaki M, Sano K, Okubo M, Yokoyama H, Ito Y, Murata I, Tsuchiya K, Minatoguchi S, Zhou X, Fujita H, Fujiwara H: Volumetric quantitative analysis of tissue characteristics of coronary plaques after statin therapy using three-dimensional integrated backscatter intravascular ultrasound. J Am Coll Cardiol 2005, 45:1946-1953.

17. Bedi U, Singh M, Singh P, Molnar J, Khosla S, Arora R: Effects of statins on progression of coronary artery disease as measured by intravascular ultrasound. J Clin Hypertens (Greenwich) 2011, 13:492-496.

18. Tian J, Gu X, Sun Y, Ban X, Xiao Y, Hu S, Yu B: Effect of statin therapy on the progression of coronary atherosclerosis. BMC Cardiovasc Disord 2012, 12:70.

19. Jadad AR, Moore RA, Carroll D, Jenkinson C, Reynolds DJ, Gavaghan DJ, McQuay HJ: Assessing the quality of reports of randomized clinical trials: is blinding necessary. Control Clin Trials 1996, 17:1-12. 
20. Schartl M, Bocksch W, Koschyk DH, Voelker W, Karsch KR, Kreuzer J, Hausmann D, Beckmann S, Gross M: Use of intravascular ultrasound to compare effects of different strategies of lipid-lowering therapy on plaque volume and composition in patients with coronary artery disease. Circulation 2001, 104:387-392.

21. Tardif JC, Gregoire J, L'Allier PL, Anderson TJ, Bertrand O, Reeves F, Title LM, Alfonso F, Schampaert E, Hassan A, McLain R, Pressler ML, Ibrahim R, Lesperance J, Blue J, Heinonen T, Rodes-Cabau J: Effects of the acyl coenzyme A: cholesterol acyltransferase inhibitor avasimibe on human atherosclerotic lesions. Circulation 2004, 110:3372-3377.

22. Nissen SE, Tuzcu EM, Brewer HB, Sipahi I, Nicholls SJ, Ganz P, Schoenhagen P. Waters DD, Pepine CJ, Crowe TD, Davidson MH, Deanfield JE, Wisniewsk LM, Hanyok JJ, Kassalow LM: Effect of ACAT inhibition on the progression of coronary atherosclerosis. N Engl J Med 2006, 354:1253-1263.

23. Nissen SE, Tardif JC, Nicholls SJ, Revkin JH, Shear CL, Duggan WT, Ruzyllo W, Bachinsky WB, Lasala GP, Tuzcu EM: Effect of torcetrapib on the progression of coronary atherosclerosis. N Engl J Med 2007, 356:1304-1316.

24. Nozue T, Yamamoto S, Tohyama S, Umezawa S, Kunishima T, Sato A, Miyake S, Takeyama Y, Morino Y, Yamauchi T, Muramatsu T, Hibi K, Sozu T, Terashima M, Michishita I: Statin treatment for coronary artery plaque composition based on intravascular ultrasound radiofrequency data analysis. Am Heart J 2012, 163:191-199. e1.

25. Zhang X, Wang H, Liu S, Gong P, Lin J, Lu J, Qiu J, Lu X: Intensive-dose atorvastatin regimen halts progression of atherosclerotic plaques in new-onset unstable angina with borderline vulnerable plaque lesions. J Cardiovasc Pharmacol Ther 2013, 18:119-125.

26. Yamada T, Azuma A, Sasaki S, Sawada T, Matsubara H: Randomized evaluation of atorvastatin in patients with coronary heart disease: a serial intravascular ultrasound study. Circ J 2007, 71:1845-1850.

27. Hong MK, Park DW, Lee CW, Lee SW, Kim YH, Kang DH, Song JK, Kim JJ, Park SW, Park SJ: Effects of statin treatments on coronary plaques assessed by volumetric virtual histology intravascular ultrasound analysis. JACC CardiovasC Interv 2009, 2:679-688.

28. Nicholls SJ, Ballantyne CM, Barter PJ, Chapman MJ, Erbel RM, Libby P, Raichlen JS, Uno K, Borgman M, Wolski K, Nissen SE: Effect of two intensive statin regimens on progression of coronary disease. N Engl J Med 2011, 365:2078-2087.

29. Lee CW, Kang SJ, Ahn JM, Song HG, Lee JY, Kim WJ, Park DW, Lee SW, Kim YH, Park SW, Park SJ: Comparison of effects of atorvastatin $(20 \mathrm{mg})$ versus rosuvastatin $(10 \mathrm{mg}$ ) therapy on mild coronary atherosclerotic plaques (from the ARTMAP trial). Am J Cardiol 2012, 109:1700-1704.

30. Hong YJ, Jeong MH, Hachinohe D, Ahmed K, Choi YH, Cho SH, Hwang SH, Ko JS, Lee MG, Park KH, Sim DS, Yoon NS, Yoon HJ, Kim KH, Park HW, Kim $J H$, Ahn Y, Cho JG, Park JC, Kang JC: Comparison of effects of rosuvastatin and atorvastatin on plaque regression in Korean patients with untreated intermediate coronary stenosis. Circ J 2011, 75:398-406.

31. Kovarnik T, Mintz GS, Skalicka H, Kral A, Horak J, Skulec R, Uhrova J, Martasek P, Downe RW, Wahle A, Sonka M, Mrazek V, Aschermann M, Linhart A: Virtual histology evaluation of atherosclerosis regression during atorvastatin and ezetimibe administration: HEAVEN study. Circ J 2012, 76:176-183.

32. Petronio AS, Amoroso G, Limbruno U, Papini B, De Carlo M, Micheli A, Ciabatti N, Mariani M: Simvastatin does not inhibit intimal hyperplasia and restenosis but promotes plaque regression in normocholesterolemic patients undergoing coronary stenting: a randomized study with intravascular ultrasound. Am Heart J 2005, 149:520-526.

33. Tani S, Watanabe I, Anazawa T, Kawamata H, Tachibana E, Furukawa K, Sato Y, Nagao K, Kanmatsuse K, Kushiro T: Effect of pravastatin on malondialdehyde-modified low-density lipoprotein levels and coronary plaque regression as determined by three-dimensional intravascular ultrasound. Am J Cardiol 2005, 96:1089-1094.

34. Rodes-Cabau J, Tardif JC, Cossette M, Bertrand OF, Ibrahim R, Larose E, Gregoire J, L'allier PL, Guertin MC: Acute effects of statin therapy on coronary atherosclerosis following an acute coronary syndrome. Am J Cardiol 2009, 104:750-757.

35. Nissen SE, Nicholls SJ, Wolski K, Rodes-Cabau J, Cannon CP, Deanfield JE, Despres JP, Kastelein JJ, SteinhubI SR, Kapadia S, Yasin M, Ruzyllo W, Gaudin C, Job B, Hu B, Bhatt DL, Lincoff AM, Tuzcu EM: Effect of rimonabant on progression of atherosclerosis in patients with abdominal obesity and coronary artery disease: the STRADIVARIUS randomized controlled trial. JAMA 2008, 299:1547-1560.
36. Nissen SE, Nicholls SJ, Wolski K, Nesto R, Kupfer S, Perez A, Jure H, De Larochelliere R, Staniloae CS, Mavromatis K, Saw J, Hu B, Lincoff AM, Tuzcu EM: Comparison of pioglitazone vs glimepiride on progression of coronary atherosclerosis in patients with type 2 diabetes: the PERISCOPE randomized controlled trial. JAMA 2008, 299:1561-1573.

37. Nissen SE, Nicholls SJ, Sipahi I, Libby P, Raichlen JS, Ballantyne CM, Davignon J, Erbel R, Fruchart JC, Tardif JC, Schoenhagen P, Crowe T, Cain V, Wolski K, Goormastic M, Tuzcu EM: Effect of very high-intensity statin therapy on regression of coronary atherosclerosis: the ASTEROID trial. JAMA 2006, 295:1556-1565.

38. Matsuzaki M, Hiramori K, Imaizumi T, Kitabatake A, Hishida H, Nomura M, Fujii T, Sakuma I, Fukami K, Honda T, Ogawa H, Yamagishi M: Intravascular ultrasound evaluation of coronary plaque regression by low density lipoprotein-apheresis in familial hypercholesterolemia: the Low Density Lipoprotein-Apheresis Coronary Morphology and Reserve Trial (LACMART). J Am Coll Cardiol 2002, 40:220-227.

39. Jensen LO, Thayssen P, Pedersen KE, Stender S, Haghfelt T: Regression of coronary atherosclerosis by simvastatin: a serial intravascular ultrasound study. Circulation 2004, 110:265-270.

40. Takayama T, Hiro T, Yamagishi M, Daida H, Hirayama A, Saito S, Yamaguchi T, Matsuzaki M: Effect of rosuvastatin on coronary atheroma in stable coronary artery disease: multicenter coronary atherosclerosis study measuring effects of rosuvastatin using intravascular ultrasound in Japanese subjects (COSMOS). Circ J 2009, 73:2110-2117.

41. Nasu K, Tsuchikane E, Katoh O, Tanaka N, Kimura M, Ehara M, Kinoshita Y, Matsubara T, Matsuo H, Asakura K, Asakura Y, Terashima M, Takayama T, Honye J, Hirayama A, Saito S, Suzuki T: Effect of fluvastatin on progression of coronary atherosclerotic plaque evaluated by virtual histology intravascular ultrasound. JACC Cardiovasc Interv 2009, 2:689-696.

42. Hattori K, Ozaki Y, Ismail TF, Okumura M, Naruse H, Kan S, Ishikawa M, Kawai T, Ohta M, Kawai H, Hashimoto T, Takagi Y, Ishii J, Serruys PW, Narula J: Impact of statin therapy on plaque characteristics as assessed by serial OCT, grayscale and integrated backscatter-IVUS. JACC CardiovasC Imaging 2012, 5:169-177.

43. Grundy SM, Cleeman JI, Merz CN, Brewer HB Jr, Clark LT, Hunninghake DB, Pasternak RC, Smith SC Jr, Stone NJ: Implications of recent clinical trials for the National Cholesterol Education Program Adult Treatment Panel III guidelines. Circulation 2004, 110:227-239.

44. Baigent C, Keech A, Kearney PM, Blackwell L, Buck G, Pollicino C, Kirby A, Sourjina T, Peto R, Collins R, Simes R: Efficacy and safety of cholesterollowering treatment: prospective meta-analysis of data from 90,056 participants in 14 randomised trials of statins. Lancet 2005, 366:1267-1278.

45. Stone NJ, Robinson J, Lichtenstein AH, Merz CN, Blum CB, Eckel RH, Goldberg AC, Gordon D, Levy D, Lloyd-Jones DM, McBride P, Schwartz JS, Shero ST, Smith SC Jr, Watson K, Wilson PW: 2013 ACC/AHA Guideline on the Treatment of Blood Cholesterol to Reduce Atherosclerotic Cardiovascular Risk in Adults: A Report of the American College of Cardiology/American Heart Association Task Force on Practice Guidelines. Circulation 2013, 00:000.

46. Hirohata A, Yamamoto K, Miyoshi T, Hatanaka K, Hirohata S, Yamawaki H, Komatsubara I, Hirose E, Kobayashi Y, Ohkawa K, Ohara M, Takafuji H, Sano F, Toyama Y, Kusachi S, Ohe T, Ito H: Four-year clinical outcomes of the OLIVUS-Ex (impact of Olmesartan on progression of coronary atherosclerosis: evaluation by intravascular ultrasound) extension trial. Atherosclerosis 2012, 220:134-138.

47. D'Ascenzo F, Agostoni P, Abbate A, Castagno D, Lipinski MJ, Vetrovec GW Frati G, Presutti DG, Quadri G, Moretti C, Gaita F, Zoccai GB: Atherosclerotic coronary plaque regression and the risk of adverse cardiovascular events: a meta-regression of randomized clinical trials. Atherosclerosis 2013, 226:178-185

48. Nissen SE, Tuzcu EM, Libby P, Thompson PD, Ghali M, Garza D, Berman L, Shi H, Buebendorf E, Topol EJ: Effect of antihypertensive agents on cardiovascular events in patients with coronary disease and normal blood pressure: the CAMELOT study: a randomized controlled trial. JAMA 2004, 292:2217-2225.

49. Hirohata A, Yamamoto K, Miyoshi T, Hatanaka K, Hirohata S, Yamawaki H, Komatsubara I, Murakami M, Hirose E, Sato S, Ohkawa K, Ishizawa M, Yamaji $\mathrm{H}$, Kawamura H, Kusachi S, Murakami T, Hina K, Ohe T: Impact of olmesartan on progression of coronary atherosclerosis a serial volumetric intravascular ultrasound analysis from the OLIVUS (impact of OLmesarten on progression of coronary atherosclerosis: evaluation by intravascular ultrasound) trial. J Am Coll Cardiol 2010, 55:976-982. 
50. Redgrave JN, Lovett JK, Rothwell PM: Histological features of symptomatic carotid plaques in relation to age and smoking: the oxford plaque study. Stroke 2010, 41:2288-2294.

51. Heidland UE, Strauer BE: Left ventricular muscle mass and elevated heart rate are associated with coronary plaque disruption. Circulation 2001, 104:1477-1482.

52. Sipahi I, Tuzcu EM, Wolski KE, Nicholls SJ, Schoenhagen P, Hu B, Balog C, Shishehbor M, Magyar WA, Crowe TD, Kapadia S, Nissen SE: Beta-blockers and progression of coronary atherosclerosis: pooled analysis of 4 intravascular ultrasonography trials. Ann Intern Med 2007, 147:10-18.

doi:10.1186/1471-2261-14-60

Cite this article as: Gao et al:: Systematic study of the effects of lowering low-density lipoprotein-cholesterol on regression of coronary atherosclerotic plaques using intravascular ultrasound. BMC Cardiovascular Disorders 2014 14:60.

\section{Submit your next manuscript to BioMed Central and take full advantage of:}

- Convenient online submission

- Thorough peer review

- No space constraints or color figure charges

- Immediate publication on acceptance

- Inclusion in PubMed, CAS, Scopus and Google Scholar

- Research which is freely available for redistribution 